\title{
EMBEDDEDNESS, CONVEXITY, AND RIGIDITY OF HYPERSURFACES IN PRODUCT SPACES
}

\author{
RONALDO FREIRE DE LIMA
}

\begin{abstract}
We establish the following Hadamard-Stoker type theorem: Let $f: M^{n} \rightarrow \mathscr{H}^{n} \times \mathbb{R}$ be a complete connected hypersurface with positive definite second fundamental form, where $\mathscr{H}^{n}$ is a Hadamard manifold. If the height function of $f$ has a critical point, then it is an embedding and $M$ is homeomorphic to $\mathbb{S}^{n}$ or $\mathbb{R}^{n}$. Furthermore, $f(M)$ bounds a convex set in $\mathscr{H}^{n} \times \mathbb{R}$. In addition, it is shown that, except for the assumption on convexity, this result is valid for hypersurfaces in $\mathbb{S}^{n} \times \mathbb{R}$ as well. We apply these theorems to show that a compact connected hypersurface in $\mathbb{Q}_{\epsilon}^{n} \times \mathbb{R}(\epsilon= \pm 1)$ is a rotational sphere, provided it has either constant mean curvature and positive-definite second fundamental form or constant sectional curvature greater than $(\epsilon+1) / 2$. We also prove that, for $\bar{M}=\mathscr{H}^{n}$ or $\mathbb{S}^{n}$, any connected proper hypersurface $f: M^{n} \rightarrow \bar{M}^{n} \times \mathbb{R}$ with positive semi-definite second fundamental form and height function with no critical points is embedded and isometric to $\Sigma^{n-1} \times \mathbb{R}$, where $\Sigma^{n-1} \subset \bar{M}^{n}$ is convex and homeomorphic to $\mathbb{S}^{n-1}$ (for $\bar{M}^{n}=\mathscr{H}^{n}$ we assume further that $f$ is cylindrically bounded). Analogous theorems for hypersurfaces in warped product spaces $\mathbb{R} \times \varrho \mathscr{H}^{n}$ and $\mathbb{R} \times \varrho \mathbb{S}^{n}$ are obtained. In all of these results, the manifold $M^{n}$ is assumed to have dimension $n \geq 3$.
\end{abstract}

\section{INTRODUCTION}

In what concerns embeddedness and convexity of surfaces in Euclidean space, one of the most fundamental results is the so-called Hadamard-Stoker Theorem. It states that a complete and positively curved surface $S$ immersed in Euclidean space is embedded, bounds an open convex set, and is homeomorphic to a sphere or a plane. J. Hadamard [17 proved it, partially, assuming $S$ compact. Subsequently, J. Stoker 33 established the complete case and showed that $S$, if noncompact, is a graph over a planar domain.

In this context, another classical result is the celebrated Cohn-Vossen Rigidity Theorem, according to which a compact surface of positive curvature in Euclidean space is rigid, that is, unique up to Euclidean rigid motions. (More generally, an isometric immersion $f: M^{n} \rightarrow \bar{M}^{n+p}$ is called rigid if, for any other isometric immersion $g: M^{n} \rightarrow \bar{M}^{n+p}$, there is an ambient isometry $\Phi: \bar{M} \rightarrow \bar{M}$ such that $g=\Phi \circ f$. If so, $f$ and $g$ are said to be congruent.)

In [30, 31, R. Sacksteder extended both the Hadamard-Stoker Theorem and the Cohn-Vossen Rigidity Theorem to nonflat hypersurfaces $f: M^{n} \rightarrow \mathbb{R}^{n+1}$ with nonnegative sectional curvature. Motivated by these results, M. do Carmo and

Departamento de Matemática - UFRN, Lagoa Nova - 59.072-970 - Brasil.

E-mail address: ronaldo@ccet.ufrn.br.

2010 Mathematics Subject Classification. 53B25 (primary), 53C24, 53C42 (secondary).

Key words and phrases. hypersurface - embeddedness - convexity - rigidity. 
F. Warner [13 considered hypersurfaces in spherical and hyperbolic space forms, obtaining then the following theorem.

Theorem (do Carmo-Warner [13). Let $f: M^{n} \rightarrow \mathbb{S}^{n+1}(n \geq 2)$ be a nontotally geodesic hypersurface, where $M$ is a compact, connected, and orientable Riemannian manifold with sectional curvature $K \geq 1$. Then, the following hold:

a) $f$ is an embedding, and $M$ is homeomorphic to $\mathbb{S}^{n}$.

b) $f(M)$ bounds a closed convex set contained in an open hemisphere of $\mathbb{S}^{n+1}$.

c) $f$ is rigid.

Moreover, the assertion (a) and the convexity property in (b) still hold if one replaces the sphere $\mathbb{S}^{n+1}$ by the hyperbolic space $\mathbb{H}^{n+1}$, and assume that $K \geq-1$.

We add that the rigidity of compact hypersurfaces $f: M^{n} \rightarrow \mathbb{H}^{n+1}$ with sectional curvature $K \geq-1$ was conjectured by do Carmo and Warner and settled affirmatively by the author and R. L. de Andrade in [11.

The conditions on the sectional curvature $K$ of $M$ in each case of do CarmoWarner Theorem, spherical and hyperbolic, can be unified by stating that the second fundamental form of the hypersurface is semi-definite, that is, its extrinsic curvature is nonnegative (see Section 2.1). Under the stronger condition of positive semi-definiteness of the second fundamental form (see [13, Remark 2.4-(b)]), S. Alexander established the following Hadamard-Stoker type theorem for compact hypersurfaces in Hadamard manifolds.

Theorem (Alexander [3). Let $f: M^{n} \rightarrow \mathscr{H}^{n+1}(n \geq 2)$ be a compact, connected, and oriented hypersurface in a Hadamard manifold $\mathscr{H}^{n+1}$. If the second fundamental form of $f$ is positive semi-definite, then $f$ is an embedding, $M$ is homeomorphic to $\mathbb{S}^{n}$, and $f(M)$ bounds an open convex set in $\mathscr{H}^{n+1}$.

Recently, some authors (see, for example, [14, 15, 16, 25]) have extended the Hadamard-Stoker Theorem to the context of hypersurfaces in products $\bar{M} \times \mathbb{R}$, giving particular attention to the cases where $\bar{M}$ is one of the non flat simply connected space forms. Considering all these results, a natural question (raised in [25]) is whether there exist Hadamard-Stoker type theorems for hypersurfaces in $\mathscr{H}^{n} \times \mathbb{R}$, where $\mathscr{H}$ (here and elsewhere) denotes a general Hadamard manifold. In this paper, we give it an affirmative answer.

We shall also focus on rigidity of hypersurfaces in $\mathscr{H}^{n} \times \mathbb{R}$ and $\mathbb{S}^{n} \times \mathbb{R}$. This, however, turns out to be a delicate matter, since these spaces have nonconstant sectional curvature. For instance, in contrast to the behavior of hypersurfaces in space forms, two hypersurfaces of either $\mathbb{H}^{n} \times \mathbb{R}$ or $\mathbb{S}^{n} \times \mathbb{R}$ with equal shape operators are not necessarily congruent. As proved by B. Daniel [10, in order to have congruence in this case, one has to ensure further that the height and angle functions of the hypersurfaces coincide (see Section 2.1 for definitions).

Taking the above considerations into account, we will investigate the rigidity of a given hypersurface $f: M^{n} \rightarrow \bar{M}^{n+1}$ in the more restricted class $\mathscr{C}_{\text {ext }}(f)$ of hypersurfaces $g: M^{n} \rightarrow \bar{M}^{n+1}$ whose extrinsic curvature coincides with the extrinsic curvature of $f$ everywhere on $M$ (cf. [29, Theorem A]). More precisely, we will say that $f$ is rigid in $\mathscr{C}_{\text {ext }}(f)$ if, for any hypersurface $g \in \mathscr{C}_{\text {ext }}(f)$, there exists an isometry $\Phi: \bar{M} \rightarrow \bar{M}$ such that $g=\Phi \circ f$. We point out that, when $\bar{M}^{n+1}$ has constant sectional curvature, the concepts of rigidity and rigidity in $\mathscr{C}_{\text {ext }}($.$) are the same.$ 
Our first result, as stated below, includes a Hadamard-Stoker type theorem for hypersurfaces in $\mathscr{H}^{n} \times \mathbb{R}$, and also a rigidity theorem for hypersurfaces in $\mathbb{H}^{n} \times \mathbb{R}$.

Theorem 1. Let $f: M^{n} \rightarrow \mathscr{H}^{n} \times \mathbb{R}(n \geq 3)$ be a complete connected oriented hypersurface with positive definite second fundamental form. If the height function of $f$ has a critical point, then the following statements hold:

a) $f$ is a proper embedding, and $M$ is either homeomorphic to $\mathbb{S}^{n}$ or $\mathbb{R}^{n}$. In the latter case, $f(M)$ is an unbounded geodesic graph over an open set of a horizontal section of $\mathscr{H}^{n} \times \mathbb{R}$.

b) $f(M)$ is the boundary of a convex set in $\mathscr{H}^{n} \times \mathbb{R}$.

c) $f$ is rigid in the class $\mathscr{C}_{\text {ext }}(f)$ when $\mathscr{H}^{n}$ is the hyperbolic space $\mathbb{H}^{n}$.

Some of the assertions of Theorem 1 remain true after replacing the Hadamard manifold $\mathscr{H}^{n}$ by the unit sphere $\mathbb{S}^{n}$. More precisely, we have the following result.

Theorem 2. Let $f: M^{n} \rightarrow \mathbb{S}^{n} \times \mathbb{R}(n \geq 3)$ be a complete connected oriented hypersurface with positive definite second fundamental form. If the height function of $f$ has a critical point, then the following statements hold:

a) $f$ is an embedding and $M$ is homeomorphic to $\mathbb{S}^{n}$ or $\mathbb{R}^{n}$. In the latter case, the height function of $f$ is unbounded.

b) $f$ is rigid in $\mathscr{C}_{\text {ext }}(f)$.

Let $\mathbb{Q}_{\epsilon}^{n}$ be $\mathbb{H}^{n}(\epsilon=-1)$ or $\mathbb{S}^{n}(\epsilon=1)$. As a consequence of Theorems 1 and 2 , we have the following extensions of two classical results on surfaces in $\mathbb{R}^{3}$.

Corollary 1 (Jellett-Liebmann type theorem). For $n \geq 3$ and $\epsilon \in\{-1,1\}$, any compact connected constant mean curvature hypersurface $f: M^{n} \rightarrow \mathbb{Q}_{\epsilon}^{n} \times \mathbb{R}$ with positive extrinsic curvature is congruent to an embedded rotational sphere.

Corollary 2 (Hilbert-Liebmann type theorem). Let $M_{c}^{n}$ be a complete, connected and orientable $n(\geq 3)$-dimensional Riemannian manifold with constant sectional curvature c. Given an isometric immersion $f: M_{c}^{n} \rightarrow \mathbb{Q}_{\epsilon}^{n} \times \mathbb{R}, \epsilon \in\{-1,1\}$, assume that $c>(1+\epsilon) / 2$. Then, $f$ is congruent to an embedded rotational sphere.

Regarding Corollary 2, we should mention that a more general result was obtained by Manfio and Tojeiro in [23, where they classify the hypersurfaces of constant sectional curvature in $\mathbb{Q}_{\epsilon}^{n} \times \mathbb{R}, n \geq 3$.

Next, we consider the dual case of the above theorems in which the height function of the hypersurface $f$ has no critical points. The condition on the second fundamental form is weakened by assuming it positive semi-definite instead of positive definite. On the other hand, $f$ is assumed to be a proper immersion, instead of complete. The precise statement is as follows.

Theorem 3. Let $f: M^{n} \rightarrow \bar{M}^{n} \times \mathbb{R}(n \geq 3)$ be a proper connected orientable hypersurface with positive semi-definite second fundamental form, where $\bar{M}^{n}$ is either a Hadamard manifold $\mathscr{H}^{n}$ or the sphere $\mathbb{S}^{n}$. Assume that:

a) The height function of $f$ has no critical points.

b) $f$ is cylindrically bounded if $\bar{M}=\mathscr{H}^{n}$, that is, there exists a closed geodesic ball $B \subset \mathscr{H}^{n}$ such that $f(M) \subset B \times \mathbb{R}$.

Then, $f$ is an embedding, and $f(M)=\Sigma \times \mathbb{R}$, where $\Sigma \subset \bar{M}^{n} \times\{0\}$ is a submanifold homeomorphic to $\mathbb{S}^{n-1}$ which bounds an open convex set in $\bar{M}^{n} \times\{0\}$. 
It should be observed that the assumption (b) in the above theorem is necessary (cf. Remark 2). It is also worth mentioning that, in the proof of the spherical case $\bar{M}=\mathbb{S}^{n}$, we apply the celebrated Soul Theorem, due to G. Perelman.

In 8 , R. Currier obtained a Hadamard-Stoker type theorem for complete connected hypersurfaces $f: M^{n} \rightarrow \mathbb{H}^{n+1}$ in hyperbolic space. He proved that, for such an $f, M$ is either homeomorphic to $\mathbb{S}^{n}$ or $\mathbb{R}^{n}$, provided all eigenvalues of its shape operator, at any point, are at least 1 . As shown by the cylinders of $\mathbb{H}^{n+1}$ of constant mean curvature, this hypothesis on the eigenvalues cannot be replaced by the weaker assumption of positive definiteness of the second fundamental form.

Currier's result can be viewed from a more general perspective if we consider $\mathbb{H}^{n+1}$ as the warped product $\mathbb{R} \times e_{e^{t}} \mathbb{R}^{n}$. In this representation, any vertical section $\{t\} \times e_{e^{t}} \mathbb{R}^{n}$ is a constant mean curvature 1 horosphere of $\mathbb{H}^{n+1}$. Therefore, for a given hypersurface $f: M \rightarrow \mathbb{R} \times e^{t} \mathbb{R}^{n}$, the main hypothesis of Currier's Theorem can be reinterpreted by saying that the eigenvalues of the shape operator of $f$ at a point $x \in M$ are all greater than, or equal to, the mean curvature of the vertical section which contains $f(x)$.

Driven by these considerations, we obtained a Hadamard-Stoker type theorem for a class of hypersurfaces (which we call $\phi$-convex) in warped products $\mathbb{R} \times{ }_{\varrho} \bar{M}^{n}$, where $\bar{M}^{n}$ is either a Hadamard manifold or the sphere $\mathbb{S}^{n}$. In these spaces, the vertical sections $\{t\} \times_{\varrho} \bar{M}^{n}$ are totally umbilical and, if properly oriented, have constant mean curvature $|\phi(t)|$, where $\phi(t)=\varrho^{\prime}(t) / \varrho(t)$. In this setting, we say that a hypersurface in $\mathbb{R} \times_{\varrho} \bar{M}^{n}$ is $\phi$-convex if, at any point, all the eigenvalues of its shape operator are bounded bellow by $|\phi|$ (see Section 4). The result, then, reads as follows.

Theorem 4. Let $\mathbb{R} \times_{\varrho} \bar{M}^{n}(n \geq 3)$ be a warped product, where $\bar{M}^{n}$ is either a Hadamard manifold or the unit sphere $\mathbb{S}^{n}$. Consider a connected, complete, and oriented strictly $\phi$-convex hypersurface $f: M^{n} \rightarrow \mathbb{R} \times_{\varrho} \bar{M}^{n}$, and assume that its height function has a critical point. Then, $f$ is a proper embedding and $M$ is either homeomorphic to $\mathbb{S}^{n}$ or $\mathbb{R}^{n}$. In the latter case, if $\bar{M}=\mathbb{S}^{n}$, the height function of $f$ is unbounded.

We remark that, when the warping function $\varrho$ is constant, strict $\phi$-convexity is equivalent to definiteness of the second fundamental form. In this manner, we can say that Theorem 4 is an extension of Theorems 1.(a) and 2.(a). Also, in the case $\bar{M}=\mathscr{H}^{n}$ of Theorem 4 , if $\varrho$ is convex, then $f$ behaves as in Theorem 1 . Namely, $f(M)$ bounds a convex set and, when $M$ is noncompact, is an unbounded geodesic graph (see Remark 4). By the same token, our final result extends the spherical case of Theorem 3 to proper $\phi$-convex hypersurfaces in $\mathbb{R} \times \varrho \mathbb{S}^{n}$ whose height function has no critical points.

Theorem 5. Assume that there exists a proper, connected, and oriented $\phi$-convex hypersurface $f: M^{n} \rightarrow \mathbb{R} \times{ }_{\varrho} \mathbb{S}^{n}(n \geq 3)$ whose height function has no critical points. Then, $f$ is an embedding with unbounded height function (above and below), and $M$ is homeomorphic to the product $\mathbb{R} \times \mathbb{S}^{n-1}$. Assuming, in addition, that $M$ has nonnegative sectional curvature, the following hold:

- $\mathrm{L}(\varrho) \leq 0$ on $\mathbb{R}$, where $\mathrm{L}(\varrho):=\left(\varrho^{\prime}\right)^{2}-\varrho \varrho^{\prime \prime}$.

- $\mathrm{L}(\varrho)=0$ on $\mathbb{R}$ if and only if $\varrho$ is constant. 
The paper is organized as follows. In Section 2, we introduce some notation and quote some results which will be used afterwards. In Section 3, we prove Theorems 113 and Corollaries 1 and 2 as well. Finally, in Section 4 after providing some background on hypersurfaces in warped products, we prove Theorems 4 and 5 .

\section{Preliminaries}

Throughout this paper, all manifolds are assumed to be $C^{\infty}$. For a given manifold $M$, we will write $T M$ for its tangent bundle. The simply connected space form of constant sectional curvature $\epsilon \in\{-1,0,1\}$ and dimension $n \geq 2$ will be denoted by $Q_{\epsilon}^{n}$, so $Q_{-1}^{n}$ is the hyperbolic space $\mathbb{H}^{n}, Q_{0}^{n}$ is the Euclidean space $\mathbb{R}^{n}$, and $Q_{1}^{n}$ is the unit sphere $\mathbb{S}^{n}$.

Recall that a complete simply connected Riemannian manifold with non-positive sectional curvature is called a Hadamard manifold. Any Hadamard manifold $\mathscr{H}^{n}$ is diffeomorphic to $\mathbb{R}^{n}$ through the exponential map. So, given $p, q \in \mathscr{H}^{n}$, there exists a unique geodesic $\gamma_{p q}$ joining these two points. A set $\Lambda \subset \mathscr{H}^{n}$ is said to be convex if $\gamma_{p q} \subset \Lambda$ whenever $p, q \in \Lambda$.

Given an $n(\geq 2)$-dimensional Riemannian manifold $\bar{M}^{n}$, consider the product $\bar{M}^{n} \times \mathbb{R}$ endowed with the standard Riemannian product metric. For a tangent vector field $Z$ in $T(\bar{M} \times \mathbb{R})=T \bar{M} \oplus T \mathbb{R}$, we will write

$$
Z=Z_{h}+Z_{v}, Z_{h} \in T \bar{M}, Z_{v} \in T \mathbb{R},
$$

and call $Z_{h}$ and $Z_{v}$ the horizontal component and the vertical component of $Z$, respectively. The projections of $\bar{M}^{n} \times \mathbb{R}$ onto its first and second factors will be denoted by $\pi_{\bar{M}}$ and $\pi_{\mathbb{R}}$, respectively, being $\pi_{\mathbb{R}}$ called the height function of $\bar{M}^{n} \times \mathbb{R}$. The gradient of $\pi_{\mathbb{R}}$, which is a parallel field in $\bar{M}^{n} \times \mathbb{R}$, will be denoted by $\partial_{t}$.

Given $t \in \mathbb{R}$, the submanifold

$$
\bar{M}_{t}:=\bar{M}^{n} \times\{t\} \subset \bar{M}^{n} \times \mathbb{R}
$$

will be called the horizontal section of $\bar{M}^{n} \times \mathbb{R}$ at level $t$. It is easily seen that horizontal sections are totally geodesic submanifolds of $\bar{M}^{n} \times \mathbb{R}$, and that each of them is isometric to $\bar{M}^{n}$. For this reason, we identify the Riemannian connection of any horizontal section $\bar{M}_{t}$ with that of $\bar{M}$ and denote it by $\bar{\nabla}$. Geodesics of $\bar{M}^{n} \times \mathbb{R}$ contained in a horizontal section will be called horizontal, whereas the ones tangent to $\partial_{t}$ will be called vertical.

\subsection{Hypersurfaces in product spaces. Given an oriented hypersurface}

$$
f: M^{n} \rightarrow \bar{M}^{n} \times \mathbb{R}
$$

we will denote its unit normal field by $N$, its second fundamental form by $\alpha$, and its shape operator by $A$. So, one has the equalities

$$
\langle\alpha(X, Y), N\rangle=\langle A X, Y\rangle=-\left\langle\widetilde{\nabla}_{X} N, Y\right\rangle=\left\langle\widetilde{\nabla}_{X} Y, N\right\rangle \forall X, Y \in T M,
$$

where $\langle$,$\rangle and \widetilde{\nabla}$ stand for the Riemannian metric and Levi-Civita connection of $\bar{M}^{n} \times \mathbb{R}$, respectively. The height function $\xi$ and the angle function $\Theta$ of $f$ are defined by the following identities:

$$
\xi(x)=\pi_{\mathbb{R}} \circ f(x) \text { and } \Theta(x)=\left\langle N(x), \partial_{t}\right\rangle, x \in M .
$$


We shall denote the gradient field and the Hessian form of a function $\zeta$ on $M$ by $\operatorname{grad} \zeta$ and Hess $\zeta$, respectively. In particular,

$$
\operatorname{grad} \xi=\partial_{t}-\Theta N \text {. }
$$

This last equality then yields

$$
x \in M \text { is a critical point of } \xi \Leftrightarrow N(x)= \pm \partial_{t} \Leftrightarrow \Theta(x)= \pm 1 .
$$

From (1), we also have that $\widetilde{\nabla}_{X} \operatorname{grad} \xi=-\Theta \widetilde{\nabla}_{X} N-X(\Theta) N$. Consequently,

$$
\operatorname{Hess} \xi(X, Y)=\Theta\langle\alpha(X, Y), N\rangle \forall X, Y \in T M \text {. }
$$

Given an open set $\Omega \subset M$ without critical points of $\xi$, a trajectory of $\operatorname{grad} \xi$ in $\Omega$ is, by definition, a curve $\varphi: I \subset \mathbb{R} \rightarrow \Omega$ which satisfies

$$
\varphi^{\prime}(s)=\operatorname{grad} \xi(\varphi(s)) \forall s \in I .
$$

It can be easily proved that, whenever $M$ is complete, one has $I=(-\infty,+\infty)$. Moreover, if the closure of $\Omega$ contains a unique critical point $x_{0}$ of $\xi$, then either

$$
\lim _{s \rightarrow-\infty} \varphi(s)=x_{0} \quad \text { or } \quad \lim _{s \rightarrow+\infty} \varphi(s)=x_{0}
$$

according as whether $x_{0}$ is a local minimum or a local maximum, respectively. In the first case, one says that the trajectory $\varphi$ is issuing from $x_{0}$, and, in the second, that $\varphi$ is going into $x_{0}$ (see [12] for details and proofs).

Concerning the gradient of $\Theta$ on $M$, for all $X \in T M$, we have

$$
X(\Theta)=X\left\langle N, \partial_{t}\right\rangle=\left\langle\widetilde{\nabla}_{X} N, \partial_{t}\right\rangle=-\left\langle A X, \partial_{t}\right\rangle=-\langle A \operatorname{grad} \xi, X\rangle .
$$

Hence, the following equality holds on $M$ :

$$
\operatorname{grad} \Theta=-A \operatorname{grad} \xi \text {. }
$$

Remark 1. When required, we will denote the second fundamental form $\alpha$ of a hypersurface $f: M \rightarrow \bar{M}^{n} \times \mathbb{R}$ by $\alpha_{f}$. The same goes for all other objects related to $f$, including its shape operator $A=A_{f}$, and its height and angle functions $\xi=\xi_{f}$, and $\Theta=\Theta_{f}$.

Consider a hypersurface $f: M \rightarrow \bar{M}^{n} \times \mathbb{R}$ and assume that a horizontal section $\bar{M}_{t}$ intersects $f(M)$ transversally. In this case, as is well known, the set $\xi^{-1}(t) \subset M$ is an $(n-1)$-dimensional submanifold of $M$. Given, then, a connected component $M_{t}$ of $\xi^{-1}(t)$, we will call the map

$$
f_{t}:=\left.f\right|_{M_{t}}: M_{t} \rightarrow \bar{M}_{t}
$$

a horizontal section of $f$ at level $t$. As unit normal field for a horizontal section $f_{t}$, we shall choose the normalized horizontal component $\eta$ of $N$, that is,

$$
\eta:=\frac{N_{h}}{\left\|N_{h}\right\|}=\frac{N-\Theta \partial_{t}}{\sqrt{1-\Theta^{2}}} .
$$

Let $u$ be a differentiable (i.e., $C^{\infty}$ ) function defined on a domain $\mathcal{D} \subset \bar{M}$. The vertical graph of $u$ in $\bar{M} \times \mathbb{R}$ is defined as the set

$$
\Sigma:=\{(p, u(p)) \in \bar{M} \times \mathbb{R} ; p \in \mathcal{D}\} .
$$

It is easily checked that $\Sigma$ is a hypersurface of $\bar{M} \times \mathbb{R}$ (seen as a submanifold). Denoting by $\nabla u$ the gradient of $u$ in $\bar{M}$ and by $\|\nabla u\|$ its norm, we have that

$$
N=\frac{-\nabla u+\partial_{t}}{\sqrt{1+\|\nabla u\|^{2}}}
$$


is clearly a unit normal field on $\Sigma$ (by abuse of notation, we are writing $\nabla u$ instead of $\left.\nabla u \circ \pi_{\bar{M}}\right)$. In particular, the angle function of $\Sigma$ is

$$
\Theta=\frac{1}{\sqrt{1+\|\nabla u\|^{2}}} \text {. }
$$

We shall denote by $\Sigma_{t}$ the level set of $u$ at $t \in u(\mathcal{D}) \subset \mathbb{R}$, that is, $\Sigma_{t}:=u^{-1}(t)$. It follows from (1) and (5) that the horizontal component of $\operatorname{grad} \xi$ on $\Sigma$ is parallel to $\nabla u$, which implies that the projection $\gamma=\pi_{\bar{M}} \circ \varphi$ of any trajectory $\varphi$ of $\operatorname{grad} \xi$ to $\bar{M}$ is tangent to $\nabla u$. Thus, such a $\gamma$ is necessarily orthogonal to all level sets $\Sigma_{t}=u^{-1}(t)$.

Let us consider now a general hypersurface $f: M^{n} \rightarrow \widetilde{M}^{n+1}$. Recall that the Gauss equation for $f$ is

(7) $\langle R(X, Y) Z, W\rangle=\langle\widetilde{R}(X, Y) Z, W\rangle+\langle\alpha(X, W), \alpha(Y, Z)\rangle-\langle\alpha(X, Z), \alpha(Y, W)\rangle$,

where $R$ and $\widetilde{R}$ are the curvature tensors of $M^{n}$ and $\widetilde{M}^{n+1}$, respectively. Denoting by $K(X, Y)$ and $\widetilde{K}(X, Y)$ the corresponding sectional curvatures of the plane generated by orthonormal vectors $X, Y \in T M$, the Gauss equation becomes

$$
K(X, Y)=\widetilde{K}(X, Y)+\langle A X, X\rangle\langle A Y, Y\rangle-\langle A X, Y\rangle^{2},
$$

where $A$ is the shape operator of $f$.

Set $\pi_{X Y}$ for the projection of $T M$ on span $\{X, Y\}$, and define the linear operator $A_{X Y}:=\left.\pi_{X Y} A\right|_{\operatorname{span}\{X, Y\}}: \operatorname{span}\{X, Y\} \rightarrow \operatorname{span}\{X, Y\}$. Then, we have

$$
\operatorname{det} A_{X Y}=\langle A X, X\rangle\langle A Y, Y\rangle-\langle A X, Y\rangle^{2} .
$$

Regarding the eigenvalues $\lambda_{1}, \ldots, \lambda_{n}$ of the shape operator $A$, it is easily shown that the following assertion holds:

$$
\lambda_{i} \geq c \geq 0 \forall i=1, \ldots, n \Rightarrow \operatorname{det} A_{X Y} \geq c^{2} \forall\{X, Y\} \text { orthonormal. }
$$

Furthermore, if the first inequality on the left is strict, so is the one on the right.

The extrinsic curvature of $f: M^{n} \rightarrow \widetilde{M}^{n+1}$ is defined by

$$
K_{\text {ext }}(f)(X, Y):=K(X, Y)-\tilde{K}(X, Y), X, Y \in T M .
$$

We shall denote by $\mathscr{C}_{\text {ext }}(f)$ the class of all hypersurfaces $g: M^{n} \rightarrow \bar{M}^{n} \times \mathbb{R}$ whose extrinsic curvature coincides with that of $f$, that is, those $g$ which satisfy:

$$
K_{\text {ext }}(f)(X, Y)=K_{\text {ext }}(g)(X, Y) \forall X, Y \in T M .
$$

Finally, we remark that, when $\widetilde{M}^{n+1}=Q_{\epsilon}^{n} \times \mathbb{R}$, the equation (8) takes the form

$$
K(X, Y)=\operatorname{det} A_{X Y}+\epsilon\left(1-\left\|\pi_{X Y} \operatorname{grad} \xi\right\|^{2}\right)
$$

(see, e.g., [10]).

2.2. Asymptotic rays in $\mathscr{H}^{n} \times \mathbb{R}$. Given a Hadamard manifold $\mathscr{H}^{n}$, we have that $\mathscr{H}^{n} \times \mathbb{R}$ is also a Hadamard manifold. Thus, we can consider the concept of asymptotic rays in this product space and profit from its properties (for details and proofs we refer the reader to [5, Section 9]).

We say that two unit speed geodesic rays $\gamma, \sigma:[0, \infty) \rightarrow \mathscr{H}^{n} \times \mathbb{R}$ are asymptotic if there is a constant $c>0$ such that $\operatorname{dist}(\gamma(s), \sigma(s)) \leq c \forall s \in[0, \infty)$, where dist stands for the distance function on $\mathscr{H}^{n} \times \mathbb{R}$.

This concept induces an equivalence relation $\sim$ in the set of all unit speed geodesic rays of $\mathscr{H}^{n} \times \mathbb{R}$. The asymptotic boundary of $\mathscr{H}^{n} \times \mathbb{R}$ is defined as the 
set of all equivalence classes determined by $\sim$. In this setting, we remark the following nice property of geodesic rays: Given $p \in \mathscr{H}^{n} \times \mathbb{R}$, and a geodesic ray $\gamma:[0, \infty) \rightarrow \mathscr{H}^{n} \times \mathbb{R}$, there exists a unique unit speed geodesic ray $\sigma_{p}$ emanating from $p$ (i.e., $\sigma_{p}(0)=p$ ) which is asymptotic to $\gamma$. Moreover, the tangent field

$$
p \in \mathscr{H}^{n} \times \mathbb{R} \mapsto \sigma_{p}^{\prime}(0) \in T_{p}\left(\mathscr{H}^{n} \times \mathbb{R}\right)
$$

is proven to be continuous (see [5, Proposition 9.6]).

The ray $\sigma_{p}$ can be constructed as follows. Take a sequence $s_{k} \rightarrow+\infty$ in $\mathbb{R}$ and, for each $k \in \mathbb{N}$, consider the geodesic $\sigma_{k}$ from $p$ to $\gamma\left(s_{k}\right)$. Then, it is shown that the sequence $\left(\sigma_{k}\right)$ converges to $\sigma_{p}$ (see [5, Proposition 9.2]).

Given a geodesic $\gamma: \mathbb{R} \rightarrow \mathscr{H}^{n} \times \mathbb{R}$, one has

$$
\frac{d}{d s}\left\langle\gamma^{\prime}(s), \partial t\right\rangle=\left\langle\widetilde{\nabla}_{\gamma^{\prime}} \gamma^{\prime}(s), \partial t\right\rangle=0
$$

that is, the angle between $\gamma^{\prime}(s)$ and $\partial_{t}$ is constant along $\gamma$. In particular, a complete geodesic of $\mathscr{H}^{n} \times \mathbb{R}$ is either horizontal or transversal to all horizontal sections.

By analogy with the idea of vertical graph, we shall employ the notion of asymptotic geodesic rays to introduce the following concept of graph in $\mathscr{H}^{n} \times \mathbb{R}$.

Definition 1. Let $U \subset \mathscr{H}_{t}$ be a subset of a horizontal section $\mathscr{H}_{t}$. We say that a set $\mathcal{G} \subset \mathscr{H}^{n} \times \mathbb{R}$ is a geodesic graph over $U$, if there exists a bijection $q \in U \leftrightarrow p=p(q) \in \mathcal{G}$ satisfying the following conditions:

- For each pair $(q, p(q)) \in U \times \mathcal{G}$, there is a geodesic ray $\sigma_{q}$ emanating from $q$ which intersects $\mathcal{G}$ only at $p$.

- For all $q, q^{\prime} \in U, \sigma_{q}$ is asymptotic to $\sigma_{q^{\prime}}$ (Fig. 1).

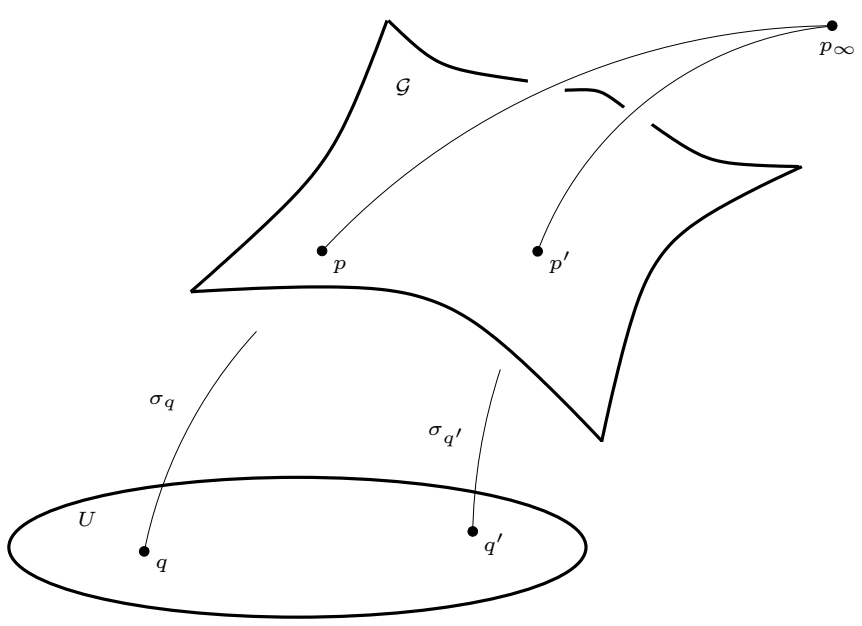

FiguRE 1. A geodesic graph in $\mathscr{H}^{n} \times \mathbb{R}$ 
2.3. Rotational spheres in $\mathbb{Q}_{\epsilon}^{n} \times \mathbb{R}$. Concluding this preliminary section, we shall briefly consider rotational hypersurfaces of $\mathbb{Q}_{\epsilon}^{n} \times \mathbb{R}$. Such a hypersurface is the orbit $\Sigma_{C}$ of a curve $C$ of $\mathbb{Q}_{\epsilon}^{n} \times \mathbb{R}$ under the action of the group of isometries of $\mathbb{Q}_{\epsilon}^{n} \times \mathbb{R}$ which fix a vertical geodesic $\{p\} \times \mathbb{R}, p \in \mathbb{Q}_{\epsilon}^{n}$. The curve $C$ is called the profile curve of $\Sigma_{C}$.

By choosing suitable profile curves, one can construct rotational hypersurfaces in $\mathbb{Q}_{\epsilon}^{n} \times \mathbb{R}$ with special properties. The general procedure is analogous to the one for the construction of the well known Delaunay surfaces, that is, the profile curve $C$ is a solution of a certain differential equation which is obtained from the conditions imposed on $\Sigma_{C}$.

With this approach, nonzero constant mean curvature (CMC, for short) rotational hypersurfaces in $\mathbb{Q}_{\epsilon}^{n} \times \mathbb{R}$ were obtained by Hsiang and Hsiang [20], for $\epsilon=-1$, and by R. Pedrosa [27, for $\epsilon=1$. Furthermore, by applying the Alexandrov reflection technique, Hsiang and Hsiang were able to prove that any compact embedded CMC hypersurface of $\mathbb{H}^{n} \times \mathbb{R}$ is spherical and rotational.

For compact embedded CMC hypersurfaces $f: M^{n} \rightarrow \mathbb{S}^{n} \times \mathbb{R}$, in general, one can apply Alexandrov reflection with respect to horizontal sections $S_{t}:=\mathbb{S}^{n} \times\{t\}$ to prove that, for some $t^{*} \in \mathbb{R}, f(M)$ is a bigraph over its projection $\pi(f(M))$ to $S_{t^{*}}$. It means that $S_{t^{*}}$ separates $f(M)$ into two symmetric connected components, and each of them is a graph over $\pi(f(M))$. If, in addition, there is an open hemisphere $\mathbb{S}_{+}^{n}$ of $\mathbb{S}^{n}$ such that $f(M) \subset S_{+}^{n} \times \mathbb{R}$, then one can perform Alexandrov reflection on "hyperplanes" $\left(\Sigma^{n-1} \cap S_{+}^{n}\right) \times \mathbb{R}$, where $\Sigma^{n-1}$ is a totally geodesic $(n-1)$-sphere of $\mathbb{S}^{n}$, and then conclude that $f$ is, in fact, rotational (see [1, Section $1-\operatorname{pg} 144$ ] and [7, Section 5]).

In [2], Aledo, Espinar and Glvez considered surfaces of $\mathbb{Q}_{\epsilon}^{2} \times \mathbb{R}$ with constant sectional curvature. They showed that, for any given $c>(\epsilon+1) / 2$, there exists a unique complete surface $f: M^{2} \rightarrow \mathbb{Q}_{\epsilon}^{2} \times \mathbb{R}$ with constant sectional curvature $c$. Such a surface is necessarily rotational and homeomorphic to $\mathbb{S}^{2}$. As mentioned in the introduction, an analogous result was obtained by Manfio and Tojeiro for hypersurfaces $f: M^{n} \rightarrow \mathbb{Q}_{\epsilon}^{n} \times \mathbb{R}(n \geq 3)$ as a consequence of their main theorems in [23. In the next section, we shall give it a new proof (cf. Corollary 22).

\section{Results on Hypersurfaces in $\mathscr{H}^{n} \times \mathbb{R}$ AND $\mathbb{S}^{n} \times \mathbb{R}$}

For the proof of assertions (a) and (b) of Theorem 1 1 , we apply Morse Theory to show that, under the given conditions, the height function of $f$ has either one critical point, and then $M$ is homeomorphic to $\mathbb{R}^{n}$, or two critical points, and then $M$ is homeomorphic to $\mathbb{S}^{n}$. In both cases, $f$ is proven to be a proper embedding by means of the Alexander Theorem [3] (see Introduction). The convexity property will be derived from a result by Bishop [4, which states that an embedded hypersurface in a Riemannian manifold with positive definite second fundamental form is strictly locally convex. Then, we apply (a) to show that, if $M$ is noncompact, then $f(M)$ is a geodesic graph in $\mathscr{H}^{n} \times \mathbb{R}$. This part of the proof is based on techniques developed by Heijenoort [19, and do Carmo and Lima [12]. Finally, the rigidity of $f$ in $\mathscr{C}_{\text {ext }}(f)$ will be obtained from B. Daniel's Fundamental Theorem [10] for hypersurfaces in $Q_{\epsilon}^{n} \times \mathbb{R}$.

First, we shall establish the following lemmas.

Lemma 1. Let $f: M^{n} \rightarrow \bar{M}^{n} \times \mathbb{R}(n \geq 3)$ be an oriented hypersurface with positive semi-definite (resp. definite) second fundamental form. Then, any horizontal 
section $f_{t}: M_{t} \rightarrow \bar{M}_{t}$ of $f$, if properly oriented, has positive semi-definite (resp. definite) second fundamental form.

Proof. Since $\bar{M}_{t}$ is totally geodesic in $\bar{M}^{n} \times \mathbb{R}$, we have that

$$
\bar{\nabla}_{X} X=\widetilde{\nabla}_{X} X \quad \forall X \in T \bar{M}_{t} .
$$

Thus, orienting $f_{t}$ as in (4), for all $X \in T M_{t}$, we have

$$
\left\langle\alpha_{f_{t}}(X, X), \eta\right\rangle=\left\langle\bar{\nabla}_{X} X, \eta\right\rangle=\frac{1}{\sqrt{1-\Theta^{2}}}\left\langle\widetilde{\nabla}_{X} X, N\right\rangle=\frac{1}{\sqrt{1-\Theta^{2}}}\left\langle\alpha_{f}(X, X), N\right\rangle \geq 0 .
$$

Hence, $\alpha_{f_{t}}$ is positive semi-definite. If $f$ has positive definite second fundamental form, the above inequality is strict, and then $f_{t}$ has positive definite second fundamental form as well.

Lemma 2. Let $f: M^{n} \rightarrow \mathbb{Q}_{\epsilon}^{n} \times \mathbb{R}(n \geq 3)$ be an oriented hypersurface whose shape operator $A_{f}$ has rank at least 3 everywhere. Then, if $g: M^{n} \rightarrow \mathbb{Q}_{\epsilon}^{n} \times \mathbb{R}$ is a hypersurface in $\mathscr{C}_{\text {ext }}(f)$ (see Section 2.1), there exists a unit normal field $N_{g} \in T M_{g}^{\perp}$ such that the corresponding shape operator $A_{g}$, the height function $\xi_{g}$, and the angle function $\Theta_{g}$ satisfy the following identities:

- $A_{f}=A_{g}$.

- $\left\|\operatorname{grad} \xi_{f}\right\|=\left\|\operatorname{grad} \xi_{g}\right\|$.

- $\Theta_{f}^{2}=\Theta_{g}^{2}$.

Proof. Since the sectional curvature determines the tensor curvature, and the extrinsic curvatures of $f$ and $g$ coincide, it follows from Gauss equation (7) that

$$
\begin{aligned}
\left\langle\alpha_{f}(X, W), \alpha_{f}(Y, Z)\right\rangle & -\left\langle\alpha_{f}(X, Z), \alpha_{f}(Y, W)\right\rangle \\
& =\left\langle\alpha_{g}(X, W), \alpha_{g}(Y, Z)\right\rangle-\left\langle\alpha_{g}(X, Z), \alpha_{g}(Y, W)\right\rangle
\end{aligned}
$$

for all $X, Y, Z, W \in T M$. Therefore, since the rank of $A_{f}$ on $M$ is at least 3, Lema 2.1 of [9] applies and gives that there exists an isometric bundle isomorphism $\mathfrak{B}: T M_{f}^{\perp} \rightarrow T M_{g}^{\perp}$ satisfying $\alpha_{g}=\mathfrak{B} \circ \alpha_{f}$. In particular, $N_{g}:=\mathfrak{B} N_{f}$ is a unit normal field to $g$. Denoting by $A_{g}$ the shape operator of $g$ with respect to $N_{g}$, for all $X, Y \in T M$, one has

$$
\left\langle A_{g} X, Y\right\rangle N_{g}=\alpha_{g}(X, Y)=\mathfrak{B} \alpha_{f}(X, Y)=\left\langle A_{f} X, Y\right\rangle \mathfrak{B} N_{f}=\left\langle A_{f} X, Y\right\rangle N_{g},
$$

which implies that $A_{f}=A_{g}$ everywhere on $M$. Considering now equality (10), we have that $\left\|\operatorname{grad} \xi_{f}\right\|=\left\|\operatorname{grad} \xi_{g}\right\|$ and, from (1), that $\Theta_{f}^{2}=\Theta_{g}^{2}$ on $M$.

Proof of Theorem 1. Let $x_{0} \in M$ be a critical point of the height function $\xi$. We can assume without loss of generality that $x_{0}$ is a local minimum, and that $\xi\left(x_{0}\right)=0$. Since the second fundamental form $\alpha$ is positive definite, equality (2) gives that Hess $\xi$ is positive definite at $x_{0}$, which implies that $x_{0}$ is a strict local minimum point of $\xi$.

Suppose that $f_{t}: M_{t} \rightarrow \mathscr{H}_{t}$ is a horizontal section of $f$ at level $t>0$. Following do Carmo and Lima [12, we say that $f_{t}$ (or, equivalently, $M_{t}$ ) is a normal section (for $x_{0}$ ) if the following conditions are satisfied:

- $M_{t}$ is homeomorphic to $\mathbb{S}^{n-1}$ and bounds an open region $\Omega_{t} \subset M$ which contains only one critical point of $\xi$; namely, $x_{0}$.

- There exists an homeomorphism $\psi: \operatorname{cl} B \rightarrow \operatorname{cl} \Omega_{t}$ such that $\psi(\partial B)=M_{t}$, where $B$ is an open ball of $\mathbb{R}^{n}$ and cl denotes closure. 
When $f_{t}$ is a normal section, we say that $t$ is a normal value and $\Omega_{t}$ is a normal region for $x_{0}$. We then write

$$
I:=\{t>0 ; t \text { is a normal value }\} \text { and } \Omega:=\bigcup \Omega_{t}, t \in I .
$$

It is clear from its definition that $\Omega$ is a nonempty open set of $M$ which is homeomorphic to $\mathbb{R}^{n}$. Setting $\partial \Omega$ for the boundary of $\Omega$, we distinguish the following mutually exclusive cases:

i) $\Omega=M$, i.e., $\partial \Omega=\emptyset$.

ii) $\Omega \neq M$ and $\partial \Omega$ contains critical points of $\xi$.

iii) $\Omega \neq M$ and $\partial \Omega$ contains no critical points of $\xi$.

By Lemma 1] each normal section $f_{t}: M_{t}^{n-1} \rightarrow \mathscr{H}_{t}^{n}$ has positive definite second fundamental form. Since we are assuming $n \geq 3$, it follows from Alexander Theorem that $f_{t}$ is an embedding and $f\left(M_{t}\right)$ bounds a compact convex set in $\mathscr{H}_{t}$. In particular, for all $t \in I,\left.f\right|_{\Omega_{t}}$ is a proper embedding. Thus, $f\left(\Omega_{t}\right)$ separates $\mathscr{H}^{n} \times[0, t)$ into two connected components, where one of them, say $\Lambda_{t}$, is bounded.

We claim that $\Lambda_{t}$ is convex. To see this, observe first that the mean curvature vector of $f$ along $\Omega_{t}$ points to $\Lambda_{t}$, that is, $\Lambda_{t}$ is the mean convex side of $\left.f\right|_{\Omega_{t}}$. Since the second fundamental form of $f$ is positive definite, a theorem by R. Bishop 4] gives that $f$ is strictly locally convex, that is, for each $x \in \Omega_{t}$, there is a neighborhood $V \subset T_{x} M$ of the null vector in the tangent space of $M$ at $x$, such that $\exp _{f(x)} V \cap \operatorname{cl} \Lambda_{t}=\{f(x)\}$. Here, exp stands for the exponential map of the ambient space $\mathscr{H}^{n} \times \mathbb{R}$.

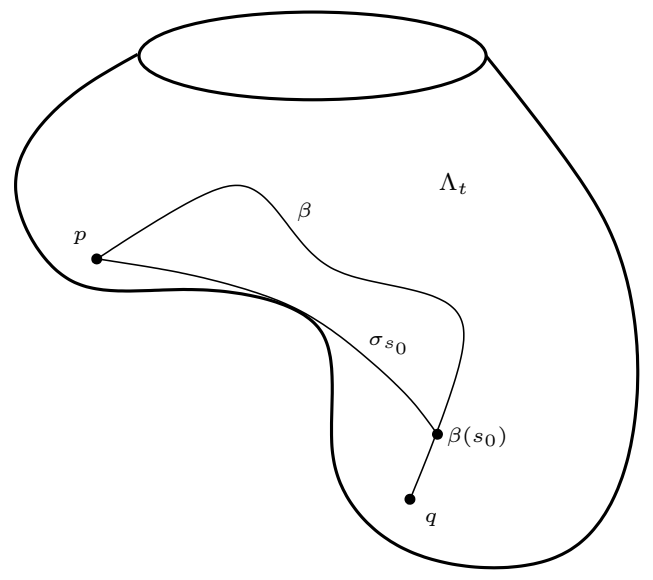

Figure 2. Proof that $\Lambda_{t}$ is convex

Suppose, for the sake of contradiction, that there are points $p, q \in \Lambda_{t}$ such that the geodesic of $\mathscr{H}^{n} \times \mathbb{R}$ which joins them is not contained in $\Lambda_{t}$. Consider, then, a curve $\beta:[0,1] \rightarrow \Lambda_{t}$ such that $\beta(0)=p$ and $\beta(1)=q$. For each $s \in(0,1]$, let $\sigma_{s}$ be the geodesic from $p$ to $\beta(s)$. For a small $s, \sigma_{s}$ is in $\Lambda_{t}$. So, there is $s_{0} \in(0,1]$ such that $\sigma_{s_{0}}$ is tangent to $f(M)$ at some point, which clearly violates the local convexity of $f$ (Fig. 2). Therefore, $\Lambda_{t}$ is convex. 
Suppose that (i) holds. In this case, $M=\Omega=\bigcup \Omega_{t}$ is homeomorphic to $\mathbb{R}^{n}$. Also, by the above considerations, $f$ is a proper embedding, and $f(M)$ is the boundary of the open convex set $\Lambda:=\bigcup \Lambda_{t}, t \in I$.

Let us prove that the interval $I$ must be unbounded if $\Omega=M$. Assuming otherwise, we have $t^{*}:=\sup I<\infty$. Hence, the horizontal section $\mathscr{H}_{t^{*}}$ is disjoint from $f(M)$, for $\xi$ has no critical points but $x_{0}$. In particular, $\mathscr{H}_{t^{*}} \subset \Lambda$. Consider an arbitrary divergent sequence $p_{k} \in \mathscr{H}_{t^{*}}, k \in \mathbb{N}$. For each $k \in \mathbb{N}$, let $\gamma_{k}$ : $\left[0, a_{k}\right] \rightarrow \Lambda$ be the unit speed geodesic of $\mathscr{H}^{n} \times \mathbb{R}$ from $f\left(x_{0}\right)$ to $p_{k}$. Passing to a subsequence, if necessary, we can assume that

$$
\gamma_{k}^{\prime}(0) \rightarrow Z_{0} \in T_{f\left(x_{0}\right)}\left(\mathscr{H}^{n} \times \mathbb{R}\right),\left\|Z_{0}\right\|=1 .
$$

Denote by $\gamma:[0,+\infty) \rightarrow \mathscr{H}^{n} \times \mathbb{R}$ the unit speed geodesic ray of $\mathscr{H}^{n} \times \mathbb{R}$ such that $\gamma(0)=f\left(x_{0}\right)$ and $\gamma^{\prime}(0)=Z_{0}$. Clearly, each geodesic segment $\gamma_{k}$ is contained in the closure of the convex set $\Lambda^{\prime} \subset \mathscr{H}^{n} \times \mathbb{R}$ bounded by $f(M)$ and $\mathscr{H}_{t^{*}}$, which implies that $\gamma \subset \operatorname{cl} \Lambda^{\prime}$. Consequently, $\gamma$ is a horizontal geodesic ray emanating from $f\left(x_{0}\right)$, i.e., it is entirely contained in $\mathscr{H}_{0}$ (otherwise, it would be transversal to $\mathscr{H}_{t^{*}}$ and would not be contained in $\left.\mathrm{cl} \Lambda^{\prime}\right)$. However, the only point of $\mathscr{H}_{0}$ in $\operatorname{cl} \Lambda^{\prime}$ is $f\left(x_{0}\right)$, which is a contradiction. Therefore, if (i) occurs, $I$ is unbounded, and so is the height function of $f$.

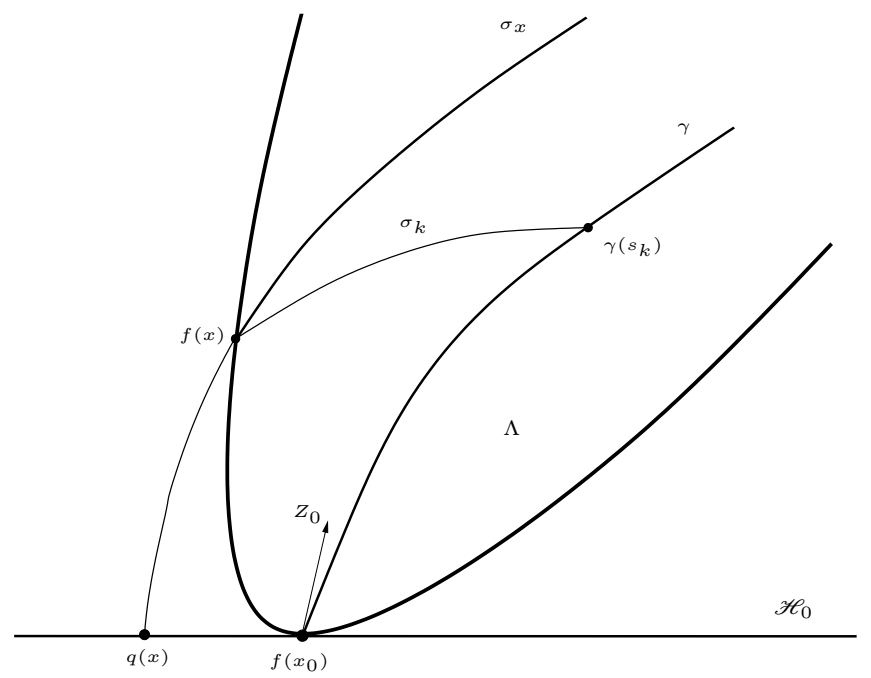

Figure 3. Proof that $f(M)$ is a geodesic graph

Still assuming (i), we shall show that $f(M)$ is a geodesic graph over an open connected set $U$ of $\mathscr{H}_{0}$. For that, consider an arbitrary divergent sequence $p_{k} \in$ $\Lambda, k \in \mathbb{N}$. Just as above, construct from that sequence a unit speed geodesic ray $\gamma:[0,+\infty) \rightarrow \mathscr{H}^{n} \times \mathbb{R}$ emanating from $f\left(x_{0}\right)$. Again, since $\Lambda$ (and so $\operatorname{cl} \Lambda$ ) is convex, $\gamma$ is contained in $\operatorname{cl} \Lambda$. Moreover, the local convexity of $f$ implies that $f\left(x_{0}\right)$ is the only point of $\gamma$ in $\operatorname{cl} \Lambda-\Lambda$.

Given $x \in M$, consider a sequence $\gamma\left(s_{k}\right)$ on $\gamma$ such that $s_{k} \rightarrow+\infty$, and let $\sigma_{k}$ be the unit speed geodesic from $f(x)$ to $\gamma\left(s_{k}\right)$. As we know, this sequence of geodesics converges to a geodesic ray $\sigma_{x}$ which is asymptotic to $\gamma$. Just as $\gamma, \sigma_{x}$ is contained in $\operatorname{cl} \Lambda$, and $f(x)$ is the only point of $\sigma_{x}$ in $\operatorname{cl} \Lambda-\Lambda$ (Fig. 3). 
Notice that $\sigma_{x}$ is not a horizontal geodesic, for the horizontal sections of $f$ are compact. Thus, the complete geodesic that contains $\sigma_{x}$ is transversal to all horizontal sections of $\mathscr{H}^{n} \times \mathbb{R}$. In particular, the geodesic ray $-\sigma_{x}$ starting at $f(x)$ in the direction $-\sigma_{x}^{\prime}(0)$ reaches $\mathscr{H}_{0}$ at some point $q=q(x)$, which implies that $f(M)$ is a geodesic graph over the set $U=\{q(x), x \in M\} \subset \mathscr{H}_{0}$. Moreover, since the exponential map and the field $x \in M \mapsto \sigma_{x}^{\prime}(0) \in T_{f(x)}\left(\mathscr{H}^{n} \times \mathbb{R}\right)$ are continuous (see Section 2.2), the map $x \in M \mapsto q(x) \in U$ is clearly a homeomorphism. In particular, $U$ is connected and open in $\mathscr{H}_{0}$.

Henceforth, we will assume that $I$ is bounded, that is, $t^{*}=\sup I<\infty$. Under this hypothesis, suppose that (ii) holds and let $x_{1} \in \partial \Omega$ be a critical point of $\xi$. Then, $\xi\left(x_{1}\right)=t^{*}$ and $N\left(x_{1}\right)= \pm \partial_{t}$. However, $N\left(x_{1}\right)=\partial_{t}$ would give that $x_{1}$ is a strict local minimum for $\xi$. In that case, there would exist a neighborhood $V$ of $x_{1}$ in $M$ such that $\left.\xi\right|_{V-\left\{x_{1}\right\}}>t^{*}$, contradicting the fact that $x_{1}$ is on the boundary of $\Omega$. Thus, $N\left(x_{1}\right)=-\partial_{t}$ and $x_{1}$ is a strict local maximum of $\xi$. In particular, $f\left(x_{1}\right)$ is isolated in $\mathscr{H}_{t^{*}}$. Consequently, in the occurrence of (ii), $M$ coincides with the closure of $\Omega$ and is, in particular, compact. Therefore, $\xi$ is a Morse function on $M$ with only two critical points, which implies that $M$ is homeomorphic to a sphere (see [24, Theorem 4.1]). The proofs that $f$ is an embedding and that $f(M)$ bounds a convex set in $\mathscr{H}^{n} \times \mathbb{R}$ are the same as in case (i) (these facts also follow from Alexander Theorem).

Finally, we shall prove that (iii) is impossible. Assume, to the contrary, that (iii) holds. In this case, $M_{t^{*}}:=\partial \Omega \subset \xi^{-1}\left(t^{*}\right)$ is a connected $(n-1)$-dimensional submanifold of $M$ which arises as the limit set of $M_{t}$ as $t \rightarrow t^{*}$. Thus, since $\left.f\right|_{M_{t}}$ is a proper embedding for all $t \in\left(0, t^{*}\right)$, the same is true for $\left.f\right|_{\mathrm{cl} \Omega}: \mathrm{cl} \Omega \rightarrow \mathscr{H}^{n} \times \mathbb{R}$. Furthermore, if we set $\Lambda=\bigcup \Lambda_{t}, t \in\left(0, t^{*}\right)$, we have that $\operatorname{cl} \Lambda$ is convex.

Suppose that $\operatorname{cl} \Lambda$ is unbounded in $\mathscr{H}^{n} \times \mathbb{R}$. Then, there exists a divergent sequence $p_{k} \in \operatorname{cl} \Lambda$. As we did before, from this sequence we obtain a geodesic ray $\gamma$ emanating from $f\left(x_{0}\right)$ which is contained in $\operatorname{cl} \Lambda$. Hence, it must be horizontal. Again, this contradicts that the only point of $\operatorname{cl} \Lambda$ on $\mathscr{H}_{0}$ is $f\left(x_{0}\right)$. Consequently, $\operatorname{cl} \Lambda$ is bounded and, therefore, compact.

Since $\left.f\right|_{\mathrm{cl} \Omega}$ is a proper embedding and $\operatorname{cl} \Lambda$ is compact, we have that $M_{t^{*}}=\partial \Omega$ is compact. Hence, for a given $t \in\left(0, t^{*}\right)$, the flow of $\operatorname{grad} \xi$ from $M_{t}$ to $M_{t^{*}}$ is a homeomorphism (see [24, Theorem 3.1]). Then, by following the trajectories of $\operatorname{grad} \xi$ through $M_{t^{*}}$, one can arrive at a normal region $M_{t^{\prime}}$ for a sufficiently small $t^{\prime}>t^{*}$, which is a contradiction. This shows the impossibility of (iii) and finishes the proof of assertions (a) and (b) of the theorem.

To prove (c), let us consider a hypersurface $g: M^{n} \rightarrow \mathbb{H}^{n} \times \mathbb{R}$ in $\mathscr{C}_{\text {ext }}(f)$. Since $\alpha_{f}$ is positive definite, we have that its shape operator has rank $n \geq 3$ everywhere. So, from Lemma 2 , with respect to a suitable normal field $N_{g} \in T M_{g}^{\perp}$, one has $A_{f}=A_{g},\left\|\operatorname{grad} \xi_{f}\right\|=\left\|\operatorname{grad} \xi_{g}\right\|$, and $\Theta_{f}^{2}=\Theta_{g}^{2}$. In particular, the set of critical points of $\xi_{f}$ and $\xi_{g}$ coincide and, then, $f$ shares with $g$ all the properties stated in (a) and (b).

Now, set $A:=A_{f}=A_{g}$, let $\Theta$ be either $\Theta_{f}$ or $\Theta_{g}$, and let $\varphi: \mathbb{R} \rightarrow M$ be a trajectory of either $\operatorname{grad} \xi_{f}$ or $\operatorname{grad} \xi_{g}$. Then, by (3),

$$
\frac{d}{d s} \Theta(\varphi(s))=\left\langle\operatorname{grad} \Theta(\varphi(s)), \varphi^{\prime}(s)\right\rangle=-\left\langle A \varphi^{\prime}(s), \varphi^{\prime}(s)\right\rangle<0,
$$

that is, the angle functions $\Theta_{f}$ and $\Theta_{g}$ are both decreasing along the trajectories of $\operatorname{grad} \xi_{f}$ and $\operatorname{grad} \xi_{g}$, respectively. Also, differentiating the equality $\Theta_{f}^{2}=\Theta_{g}^{2}$ 
and using (3), we easily conclude that

$$
\Theta_{f} \operatorname{grad} \xi_{f}=\Theta_{g} \operatorname{grad} \xi_{g} .
$$

After a possible reflection about an horizontal section of $\mathbb{H}^{n} \times \mathbb{R}$ (which is an isometry), we can assume that $x_{0} \in M$ is a minimum point of $\xi_{g}$. In this case, one has $\Theta_{f}\left(x_{0}\right)=\Theta_{g}\left(x_{0}\right)=1$. Since $\Theta_{f}= \pm \Theta_{g}$, by continuity, $\Theta_{f}=\Theta_{g}$ in a neighborhood $V$ of $x_{0}$, which gives that $\operatorname{grad} \xi_{f}=\operatorname{grad} \xi_{g}$ on $V$. Hence, on $V$, the trajectories of $\operatorname{grad} \xi_{f}$ and $\operatorname{grad} \xi_{g}$ coincide. However, $\Theta_{f}$ and $\Theta_{g}$ are both decreasing along these trajectories. Thus, the identity $\Theta_{f}=\Theta_{g}$, and so $\operatorname{grad} \xi_{f}=\operatorname{grad} \xi_{g}$, extends to all of $M$.

It follows that the equalities

$$
A_{f}=A_{g}, \quad \Theta_{f}=\Theta_{g} \quad \text { and } \quad \operatorname{grad} \xi_{f}=\operatorname{grad} \xi_{g}
$$

hold everywhere in $M$. Therefore, by Daniel Theorem [10, there exists an isometry

$$
\Phi: \mathbb{H}^{n} \times \mathbb{R} \rightarrow \mathbb{H}^{n} \times \mathbb{R}
$$

such that $g=\Phi \circ f$. This shows (c) and concludes the proof of the theorem.

Proof of Theorem 2. We just sketch the proof, since the argument is similar to the one in the proof of Theorem 1. By Lemma 1, the horizontal sections of $f$ have positive definite second fundamental form. Considering do Carmo-Warner Theorem and keeping the notation of the proof of Theorem 1, one has that all normal sections of $f$ are compact and embedded. Thus, if (i) occurs, $M$ is homeomorphic to $\mathbb{R}^{n}$ and $f$ is properly embedded. From this last property, $f(M)$ is not contained in the compact region bounded by two horizontal sections of $\mathbb{S}^{n} \times \mathbb{R}$, which implies that the the height function of $f$ is unbounded. The possibility (ii), analogously, gives that $f$ is properly embedded and that $M$ is homeomorphic to $\mathbb{S}^{n}$. The possibility (iii) is easily ruled out, for the horizontal sections of $\mathbb{S}^{n} \times \mathbb{R}$ are necessarily compact. This proves (a). Regarding (b), we have just to consider Lemma 2, and remember that Daniel Theorem [10] is set in $\mathbb{H}^{n} \times \mathbb{R}$ and $\mathbb{S}^{n} \times \mathbb{R}$ as well.

Proof of Corollary 1. Since $M$ is compact, the height function of $f$ has a critical point. Therefore, from Theorems 1 and $2, f$ is an embedding and $M$ is homeomorphic to $\mathbb{S}^{n}$. Thus, for $\epsilon=-1$, the main theorems in [20] give that $f$ is congruent to a rotational sphere of positive constant mean curvature.

Let us consider now the case $\epsilon=1$. In this setting, as discussed in Section 2.3, we can perform Alexandrov reflection with respect to horizontal sections $S_{t}=\mathbb{S}^{n} \times\{t\}$ to conclude that, for some $t^{*} \in \mathbb{R}, f(M)$ is a bigraph over its projection $\pi(f(M))$ to $S_{t^{*}}$. Thus, writing $f_{t^{*}}: M_{t^{*}} \rightarrow S_{t^{*}}$ for the horizontal section of $f$ at $t^{*}$, we have that $f\left(M_{t^{*}}\right)$ is the boundary of $\pi(f(M))$.

By Lemma 1, $f_{t^{*}}$ has positive extrinsic curvature. In particular, it is non totally geodesic. Thus, by do Carmo-Warner Theorem, $f\left(M_{t^{*}}\right)$ is contained in an open hemisphere $S_{t^{*}}^{+}$of $S_{t^{*}}$. So, the same is true for $\pi(f(M))$, that is, $f(M) \subset S_{t^{*}}^{+} \times \mathbb{R}$. As also discussed in Section 2.3, this implies that we can perform Alexandrov reflections on $f(M)$ and conclude that it is congruent to a rotational sphere of positive constant mean curvature.

Proof of Corollary 2. Since $c>(1+\epsilon) / 2 \geq 0$, by Myers Theorem, $M$ is compact. Also, it is easily seen that the maximum value of the sectional curvature in $\mathbb{Q}_{\epsilon}^{n} \times \mathbb{R}$ is $(1+\epsilon) / 2$. This, together with Gauss equation and the condition on $c$, implies 
that $f$ has positive definite second fundamental form, if properly oriented. Thus, Theorems 1 and 2 apply and give that $f$ is an isometric embedding of the standard sphere $S_{c}^{n}$ of constant sectional curvature $c$ into $\mathbb{Q}_{\epsilon}^{n} \times \mathbb{R}$. In addition, as seen in the proofs of these theorems, the height function $\xi$ of $f$ has exactly two critical points; a minimum $x_{0}$ and a maximum $x_{1}$. As before, assume $\xi\left(x_{0}\right)=0$ and observe that $\Theta\left(x_{0}\right)=1$ and $\Theta\left(x_{1}\right)=-1$.

By 23, Lemma 3.1] (see also [22, Lemma 5]), $\operatorname{grad} \xi$ is an eigenvector of the shape operator $A$ on $M-\left\{x_{0}, x_{1}\right\}$. Since tangent vectors of horizontal sections $f_{t}: M_{t} \rightarrow \mathbb{Q}_{\epsilon}^{n} \times\{t\}$ are orthogonal to $\operatorname{grad} \xi$, it follows from 3 that the angle function $\Theta$ of $f$ is constant along the horizontal sections $f_{t}$.

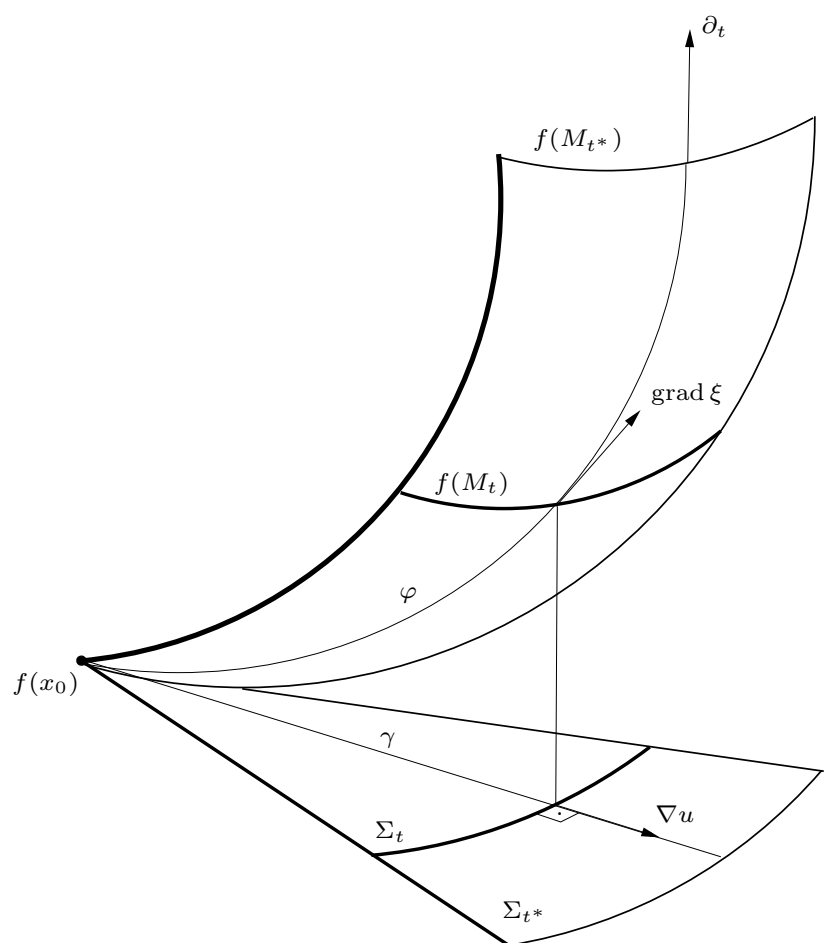

Figure 4. A piece of the rotational graph $\Sigma$

Notice that the trajectories of $\operatorname{grad} \xi$ cover $M-\left\{x_{0}, x_{1}\right\}$, and any of them issues from $x_{0}$ and goes into $x_{1}$ (cf. Section 2.1). Moreover, along these trajectories, $\Theta$ decreases from 1 to -1 (see $\sqrt{11})$ ). Therefore, for some $t^{*} \in\left(0, \xi\left(x_{1}\right)\right)$, the angle function $\Theta$ vanishes on $M_{t^{*}}$, and is positive on $M_{t}$ for $t \in\left(0, t^{*}\right)$. In particular, the set $\Sigma$ of all points of $f(M)$ at height less than $t^{*}$ is a vertical graph of a differentiable function $u$ on the projection $\mathcal{D}:=\pi(\Sigma) \subset \mathbb{Q}_{\epsilon}^{n}$. It should also be noticed that the level hypersurfaces $\Sigma_{t}:=u^{-1}(t) \subset \mathcal{D}, t \in\left(0, t^{*}\right)$, are all topological $(n-1)$-spheres.

Since $\Theta$ is constant along the horizontal sections of $f$, it follows from 6 that $\|\nabla u\|$ is constant along the level spheres $\Sigma_{t}$. Hence, any trajectory $\gamma$ of $\nabla u$ is actually a geodesic of $\mathbb{Q}_{\epsilon}^{n}$ (cf. [34, Lemma 1]). However, as we have discussed before, such a geodesic $\gamma$ is nothing but the (reparametrized) projection of a trajectory of 
$\operatorname{grad} \xi$ to $\mathcal{D}$. So, any $\gamma$ is a geodesic of $\mathbb{Q}_{\epsilon}^{n}$ emanating from $f\left(x_{0}\right)$ and orthogonal to all level spheres $\Sigma_{t}$ (Fig. 4).

We conclude from this last statement that, for all $t \in\left(0, t^{*}\right], \quad \Sigma_{t}$ is a round geodesic sphere of $\mathbb{Q}_{\epsilon}^{n}$ centered at $f\left(x_{0}\right)$. Thus, $\mathcal{D}$ is the open ball of $\mathbb{Q}_{\epsilon}^{n}$ centered at $f\left(x_{0}\right)$ and bounded by $\Sigma_{t^{*}}$, and the graph $\Sigma \subset f(M)$ is rotational with axis $f\left(x_{0}\right) \times \mathbb{R}$ and boundary $f\left(M_{t^{*}}\right)$.

An analogous reasoning applied to $\Sigma^{\prime}=f(M)-\operatorname{cl} \Sigma$ leads to the conclusion that $\Sigma^{\prime}$ is rotational with axis $f\left(x_{1}\right) \times \mathbb{R}$ and boundary $f\left(M_{t^{*}}\right)$. Since $\Sigma$ and $\Sigma^{\prime}$ are both rotational and have the geodesic $(n-1)$-sphere $f\left(M_{t^{*}}\right)$ as their common boundary, their axes must coincide. Hence, $f(M)=\operatorname{cl} \Sigma \cup \operatorname{cl} \Sigma^{\prime}$ is indeed a rotational sphere, as we wished to prove.

Recall that, as proved by Cheeger and Gromoll [6, any complete and noncompact Riemannian manifold $M$ with nonnegative sectional curvature has a compact submanifold, called the soul of $M$, whose normal bundle in $M$ is diffeomorphic to $M$. If $M$ has at least one point at which all sectional curvatures are positive, then the soul of $M$ is a single point (and then $M$ is homeomorphic to $\mathbb{R}^{n}$ ). This fact, conjectured by Cheeger and Gromoll, was proved by G. Perelman in 28, and called the Soul Theorem. In what follows, we shall give a proof of Theorem 3 in which the Soul Theorem plays a crucial role.

Proof of Theorem 3. Since $B_{t}:=B \times\{t\}$ and $S_{t}:=\mathbb{S}^{n} \times\{t\}$ are both compact, the height function $\xi$ is unbounded above and below on $M$, for $f$ is proper and $\xi$ has no critical points. Thus, setting $\bar{M}_{t}$ for $B_{t}$ or $S_{t}$, one has $M=\bigcup M_{t}$, $t \in \mathbb{R}$, where $f_{t}: M_{t} \subset M \rightarrow \bar{M}_{t}$ is a family of compact and connected horizontal sections of $f$.

By Lemma 1. the sections $f_{t}$ have positive semi-definite second fundamental form. Hence, Alexander and do Carmo-Warner Theorems apply and give that, for all $t \in \mathbb{R}, M_{t}$ is homeomorphic to $\mathbb{S}^{n-1}, f_{t}$ is an embedding, and $f\left(M_{t}\right)$ bounds a convex set in $\bar{M}_{t}$. Therefore, $f$ is an embedding and $M$ is homeomorphic to the product $\mathbb{S}^{n-1} \times \mathbb{R}$.

Suppose that $\bar{M}=\mathscr{H}^{n}$. In this case, since we are assuming the second fundamental form of $f$ positive semi-definite, we can apply Bishop Theorem as in the proof of Theorem 1, and equally conclude that the mean convex side $\Lambda \subset B \times \mathbb{R}$ of $f$ is convex (we remark that Bishop Theorem only requires semi-definiteness of the second fundamental form). Hence, given $t \in \mathbb{R}$, if we choose $x_{0} \in M_{t}$ and a divergent sequence $\left(x_{k}\right)$ in $M$ such that $\xi\left(x_{k}\right) \rightarrow \pm \infty$, each geodesic segment $\gamma_{k}$ of $\mathscr{H}^{n} \times \mathbb{R}$ from $f\left(x_{0}\right)$ to $f\left(x_{k}\right)$ is contained in $\operatorname{cl} \Lambda$. Consequently, the limit geodesic ray $\gamma=\lim \gamma_{k}$ emanating from $f\left(x_{0}\right)$ is contained in $\operatorname{cl} \Lambda$ as well. If $\gamma$ were not a vertical geodesic ray, it would eventually reach the boundary of $B \times \mathbb{R}$, which is impossible, since $\operatorname{cl} \Lambda \subset B \times \mathbb{R}$. So, $\gamma$ is vertical and tangent to $f$ at $x_{0}$, that is, $T_{x_{0}} M$ is vertical. Being both $t$ and $x_{0}$ arbitrary, it follows that $f(M)=f\left(M_{0}\right) \times \mathbb{R}$.

Let us assume now that $\bar{M}=\mathbb{S}^{n}$. Under this assumption, the sectional curvature $K$ of $M$ is nonnegative, for $\mathbb{S}^{n} \times \mathbb{R}$ has nonnegative sectional curvature and, by the hypothesis, $f$ has nonnegative extrinsic curvature. Since $M$ is noncompact, it implies that, for all $x \in M$, there exist orthonormal vectors $X, Y \in T_{x} M$ satisfying $K(X, Y)=0$ (otherwise, by the Soul Theorem, $M$ would be homeomorphic to 
$\left.\mathbb{R}^{n}\right)$. However, from implication $(9)$ and equality $(10)$,

$$
\left\|\pi_{X Y} \operatorname{grad} \xi\right\|^{2}=1+\operatorname{det} A_{X Y} \geq 1 .
$$

Thus, $\left\|\pi_{X Y} \operatorname{grad} \xi(x)\right\|=1$, i.e., $\|\operatorname{grad} \xi(x)\|=1$, and so

$$
\operatorname{grad} \xi(x)=\partial_{t} \forall x \in M,
$$

which clearly implies that $f(M)=f\left(M_{0}\right) \times \mathbb{R}$. This finishes the proof.

Remark 2. The assumption (b) in Theorem 3 is necessary. Indeed, as noted in 32 , pg. 124], there exists a proper unbounded immersion $g: M^{n-1} \rightarrow \mathbb{H}^{n}(n \geq 3)$ with positive definite second fundamental form, which is not an embedding. Therefore, on one hand, the immersion $f:=g \times$ id : $M^{n-1} \times \mathbb{R} \rightarrow \mathbb{H}^{n} \times \mathbb{R}$ is proper, has positive semi-definite second fundamental form, and its height function has no critical points. On the other hand, $f$ is not an embedding. (Notice that $f$ is non cylindrically bounded, for $g$ is unbounded.)

\section{Results on Hypersurfaces in $\mathbb{R} \times{ }_{\varrho} \mathscr{H}^{n}$ AND $\mathbb{R} \times{ }_{\varrho} \mathbb{S}^{n}$}

Given an $n$-dimensional Riemannian manifold $\bar{M}^{n}$, and a positive differentiable function $\varrho: \mathbb{R} \rightarrow \mathbb{R}$, the warped product $\mathbb{R} \times \varrho \bar{M}^{n}$ is, by definition, the manifold $\mathbb{R} \times \bar{M}^{n}$ endowed with the metric

$$
\langle X, Y\rangle=\left\langle X_{h}, Y_{h}\right\rangle_{\mathbb{R}}+\varrho^{2}(t)\left\langle X_{v}, Y_{v}\right\rangle_{\bar{M}}, X, Y \in T_{(t, p)}(\mathbb{R} \times \bar{M}),(t, p) \in \mathbb{R} \times \bar{M} .
$$

Here $\langle,\rangle_{\mathbb{R}}$ and $\langle,\rangle_{\bar{M}}$ denote the Riemannian metrics of $\mathbb{R}$ and $\bar{M}^{n}$, respectively, and the notation is as in Section 2 (notice that, now, horizontal vectors are tangent to $\mathbb{R}$, whereas vertical vectors are tangent to $\bar{M}$ ).

It is easily seen that, in $\mathbb{R} \times{ }_{\varrho} \bar{M}^{n}$, all vertical sections

$$
\bar{M}_{t}:=\{t\} \times_{\varrho} \bar{M}^{n}
$$

are homothetic to $\bar{M}$. In particular, the following hold:

- Vertical sections are Hadamard manifolds (resp. spheres with constant sectional curvature) if $\bar{M}$ is a Hadamard manifold (resp. $\mathbb{S}^{n}$ ).

- The Riemannian connection of any vertical section, to be denoted by $\bar{\nabla}$, can be identified with that of $\bar{M}$ (see [26, Lema 64, pg. 92]).

Denoting by $\widetilde{\nabla}$ the Riemannian connection of $\mathbb{R} \times \varrho \bar{M}^{n}$ and defining

$$
\phi(t):=\frac{\varrho^{\prime}(t)}{\varrho(t)}, \quad t \in \mathbb{R},
$$

for any vertical fields $X, Y \in T \bar{M}$, the following identities hold (see [5, Lema 7.3]):

$$
\begin{aligned}
\widetilde{\nabla}_{X} Y & =\bar{\nabla}_{X} Y-\phi\langle X, Y\rangle \partial_{t} . \\
\widetilde{\nabla}_{X} \partial_{t} & =\widetilde{\nabla}_{\partial_{t}} X=\phi X . \\
\widetilde{\nabla}_{\partial_{t}} \partial_{t} & =0 .
\end{aligned}
$$

Let us introduce now the concept of $\phi$-convexity of hypersurfaces in warped products $\mathbb{R} \times \varrho \bar{M}$. As we pointed out in the introduction, when $\varrho$ is constant, $\phi$-convexity is equivalent to positive semi-definiteness of the second fundamental form. 
Definition 2. An oriented hypersurface $f: M^{n} \rightarrow \mathbb{R} \times_{\varrho} \bar{M}$ is called $\phi$-convex (resp. strictly $\phi$-convex) if, for all $x \in M$, each eigenvalue $\lambda$ of its shape operator at $x$ satisfies $\lambda \geq|\phi \circ \xi(x)|$ (resp. $\lambda>|\phi \circ \xi(x)|$ ).

Vertical sections are trivial examples of $\phi$-convex hypersurfaces of $\mathbb{R} \times_{\varrho} \bar{M}^{n}$. In the case where $\bar{M}$ is a simply connected space form, they are the only $\phi$-convex hypersurfaces under certain restrictions on the warp function $\varrho$ and the immersed manifold $M$ (see Proposition 1 at the end of this section). In hyperbolic space $\mathbb{H}^{n+1}=\mathbb{R} \times e_{e^{t}} \mathbb{R}^{n}$, as we have discussed, the vertical sections are the constant mean curvature one horospheres. In particular, the geodesic spheres of $\mathbb{H}^{n+1}$ are all $\phi$ convex, since they are totally umbilical and have constant mean curvature greater than one.

Concerning oriented hypersurfaces $f: M^{n} \rightarrow \mathbb{R} \times{ }_{\varrho} \bar{M}^{n}$, we shall keep the notation of the previous sections. Namely, for such an $f, N$ will denote its unit normal field, $A$ its shape operator, $\Theta=\left\langle N, \partial_{t}\right\rangle$ its angle function, and $\xi=\pi_{\mathbb{R}} \circ f$ its height function. In particular, as before, $\operatorname{grad} \xi=\partial_{t}-\Theta N$.

Remark 3. To avoid excessive notation, we will write $\varrho$ and $\phi$ for the compositions $\varrho \circ \xi$ and $\phi \circ \xi$, respectively, since there is no danger of confusion.

From the equalities $(12)$, for all $X \in T(\mathbb{R} \times \varrho \bar{M})$, one has

$$
\widetilde{\nabla}_{X} \partial_{t}=\widetilde{\nabla}_{X_{v}} \partial_{t}=\phi X_{v}=\phi\left(X-\left\langle X, \partial_{t}\right\rangle \partial_{t}\right) .
$$

Thus, if $X \in T M$,

$$
X(\Theta)=\left\langle\widetilde{\nabla}_{X} N, \partial_{t}\right\rangle+\left\langle N, \widetilde{\nabla}_{X} \partial_{t}\right\rangle=-\langle A \operatorname{grad} \xi, X\rangle-\phi \Theta\langle\operatorname{grad} \xi, X\rangle .
$$

Hence, the gradient of $\Theta$ is

$$
\operatorname{grad} \Theta=-(A+\phi \Theta \mathrm{Id}) \operatorname{grad} \xi,
$$

where Id stands for the identity map of $T M$.

Given $X, Y \in T M$, we have that

$$
\begin{aligned}
\operatorname{Hess} \xi(X, Y) & =\left\langle\widetilde{\nabla}_{X} \operatorname{grad} \xi, Y\right\rangle=\left\langle\widetilde{\nabla}_{X_{v}} \partial_{t}, Y\right\rangle-\Theta\left\langle\widetilde{\nabla}_{X} N, Y\right\rangle \\
& =\phi\left\langle X_{v}, Y\right\rangle+\Theta\langle\alpha(X, Y), N\rangle \\
& =\phi\left(\langle X, Y\rangle-\left\langle X, \partial_{t}\right\rangle\left\langle Y, \partial_{t}\right\rangle\right)+\Theta\langle\alpha(X, Y), N\rangle .
\end{aligned}
$$

In particular,

$$
\operatorname{Hess} \xi(X, X)=\phi\left(\langle X, X\rangle-\left\langle X, \partial_{t}\right\rangle^{2}\right)+\Theta\langle\alpha(X, X), N\rangle \forall X \in T M
$$

We also call attention to the fact that, defining the L-operator

$$
\mathrm{L}(\varrho):=\left(\varrho^{\prime}\right)^{2}-\varrho \varrho^{\prime \prime},
$$

we have from Gauss equation for hypersurfaces $f: M^{n} \rightarrow \mathbb{R} \times_{\varrho} \mathbb{Q}_{\epsilon}^{n}$ (see, e.g., 21, Proposition 3]) that, for all orthonormal tangent fields $X, Y \in T M$, the sectional curvature $K$ of $M$ satisfies

$$
K(X, Y)=\left(\frac{\epsilon}{\varrho^{2}}-\phi^{2}\right)+\left(\frac{\mathrm{L}(\varrho)-\epsilon}{\varrho^{2}}\right)\left\|\pi_{X Y} \operatorname{grad} \xi\right\|^{2}+\operatorname{det} A_{X Y} .
$$

We proceed now to the proofs of Theorems 4 and 5 First, we establish the following Lemma, which can be considered as a "warped" version of Lemma 1 
Lemma 3. Let $f: M^{n} \rightarrow \mathbb{R} \times_{\varrho} \bar{M}^{n} \quad(n \geq 3)$ be a $\phi$-convex (resp. strictly $\phi$-convex) hypersurface. Then, for all $X \in T M$, one has

$$
\langle\alpha(X, X), N\rangle \pm \phi \Theta\langle X, X\rangle \geq 0 \text { (resp. }>0) .
$$

Consequently, any vertical section $f_{t}: M_{t} \rightarrow \bar{M}_{t}$ of $f$, if properly oriented, has positive semi-definite (resp. definite) second fundamental form.

Proof. Consider an orthonormal frame $\left\{X_{1}, \ldots, X_{n}\right\} \subset T M$ of eigenvectors of the shape operator $A$ of $f$ with corresponding eigenvalues $\lambda_{1}, \ldots, \lambda_{n}$. The $\phi$ convexity of $f$ yields $\lambda_{i} \geq|\phi|$ for all $i=1, \ldots, n$. Since $-1 \leq \Theta \leq 1$, by setting $X=a_{1} X_{1}+\cdots+a_{n} X_{n}$, one has

$$
\langle\alpha(X, X), N\rangle=\langle A X, X\rangle=\sum_{i=1}^{n} \lambda_{i} a_{i}^{2} \geq|\phi|\langle X, X\rangle \geq \pm \phi \Theta\langle X, X\rangle,
$$

which gives (17).

Let us consider now a vertical section $f_{t}: M_{t} \rightarrow \bar{M}_{t}$ with orientation

$$
\eta=\frac{N_{v}}{\left\|N_{v}\right\|}=\frac{1}{\sqrt{1-\Theta^{2}}}\left(N-\Theta \partial_{t}\right)
$$

In this case, for all $X \in T M_{t}$,

$$
\begin{aligned}
\left\langle\alpha_{f_{t}}(X, X), \eta\right\rangle & =\frac{1}{\sqrt{1-\Theta^{2}}}\left\langle\bar{\nabla}_{X} X, N-\Theta \partial_{t}\right\rangle=\frac{1}{\sqrt{1-\Theta^{2}}}\left\langle\bar{\nabla}_{X} X, N\right\rangle \\
& =\frac{1}{\sqrt{1-\Theta^{2}}}\left(\left\langle\widetilde{\nabla}_{X} X, N\right\rangle+\phi \Theta\langle X, X\rangle\right),
\end{aligned}
$$

where the last equality followed from the first one in 12 . This, together with inequality (17), gives

$$
\left\langle\alpha_{f_{t}}(X, X), \eta\right\rangle=\frac{1}{\sqrt{1-\Theta^{2}}}(\langle\alpha(X, X), N\rangle+\phi \Theta\langle X, X\rangle) \geq 0,
$$

which implies that $f_{t}$ has positive semi-definite second fundamental form. If $f$ is strictly $\phi$-convex, the inequality $(17)$ is strict and, then, $f_{t}$ has positive definite second fundamental form.

Proof of Theorem 4. Let $x_{0} \in M$ be a critical point of the height function $\xi$ of $f$. It follows from the Hessian formula (15) and inequality (17) that $x_{0}$ is a strict maximum if $\Theta\left(x_{0}\right)=-1$, or is a strict minimum if $\Theta\left(x_{0}\right)=1$. Let us assume the latter, and also that $\xi\left(x_{0}\right)=0$.

Define normal sections, normal regions and normal values for $x_{0}$ as in the proof of Theorem 1. As before, denote by $\Omega \subset M$ the union of all normal regions, and by $I \subset(0,+\infty)$ the interval of all normal values. Recall that $\Omega$ is homeomorphic to $\mathbb{R}^{n}$ and consider the cases:

i) $\Omega=M$.

ii) $\Omega \neq M$ and $\partial \Omega$ contains critical points of $\xi$.

iii) $\Omega \neq M$ and $\partial \Omega$ contains no critical points of $\xi$.

The vertical sections $\bar{M}_{t}$ are either all Hadamard manifolds or all spheres with constant sectional curvature. Thus, do Carmo-Warner and Alexander Theorems, together with Lemma 3 , imply that each normal section $f_{t}: M_{t} \rightarrow \bar{M}_{t}$ is an embedding. Therefore, if (i) occurs, $M$ is homeomorphic to $\mathbb{R}^{n}$ and $f$ is a proper 
embedding. In particular, if $\bar{M}=\mathbb{S}^{n}$, the height function of $f$ is unbounded, since the region bounded by two normal sections of $\mathbb{R} \times{ }_{\varrho} \mathbb{S}^{n}$ is compact.

If (ii) holds, then we can argue just as in the proof of Theorem 1 to conclude that $\xi$ has precisely two critical points, giving that $M$ is homeomorphic to $\mathbb{S}^{n}$.

Finally, let us assume that (iii) holds and then derive a contradiction. Reasoning as in the proof of Theorem 1 , it suffices to prove that $M_{t^{*}}:=\partial \Omega$ is compact, where $t^{*}=\sup I$.

As before, we have that $f_{t^{*}}: M_{t^{*}} \rightarrow \bar{M}_{t^{*}}$ is a proper embedding. If $\bar{M}^{n}=\mathbb{S}^{n}$, then $\bar{M}_{t^{*}}$ is a sphere, which implies that $M_{t^{*}}$ is compact, since $f_{t^{*}}$ is proper. Hence, (iii) does not hold if $\bar{M}^{n}=\mathbb{S}^{n}$.

Let us suppose now that $\bar{M}^{n}$ is a Hadamard manifold. Under this hypothesis, we shall prove that the projections of $f\left(\Omega_{t}\right)$ to $\bar{M}_{t^{*}}$ are uniformly bounded, which will imply that $M_{t^{*}}$ is compact. In our reasoning, we will use some ideas contained in Currier's proof of his [8, Theorem A].

Let $\pi: \mathbb{R} \times \varrho \bar{M}^{n} \rightarrow \bar{M}_{t^{*}}$ be the projection onto $\bar{M}_{t^{*}}$. Given $t$ in $\left(0, t^{*}\right)$, set $\Omega_{t}^{*}=\pi\left(f\left(\Omega_{t}\right)\right) \subset \bar{M}_{t^{*}}$, consider a boundary point $x^{*} \in \partial \Omega_{t}^{*}$, and let $x \in \operatorname{cl} \Omega_{t}$ be such that $\pi(f(x))=x^{*}$. Then, choose $t_{0} \in\left(0, t^{*}\right), t_{0}<t$, in such a way that the angle function $\Theta$ is positive in $\operatorname{cl} \Omega_{t_{0}}$ (notice that $\Theta\left(x_{0}\right)=1$ ). In this setting, we have either $x \in \partial \Omega_{t}=M_{t}$ or $x \in \Omega_{t}$. In the latter case, it is clear that the horizontal geodesic (i.e., parallel to $\partial_{t}$ ) through $f(x)$ is tangent to $M$, that is, $\Theta(x)=0$. So, in any case, $x \notin \mathrm{cl} \Omega_{t_{0}}$ (Fig. 5).

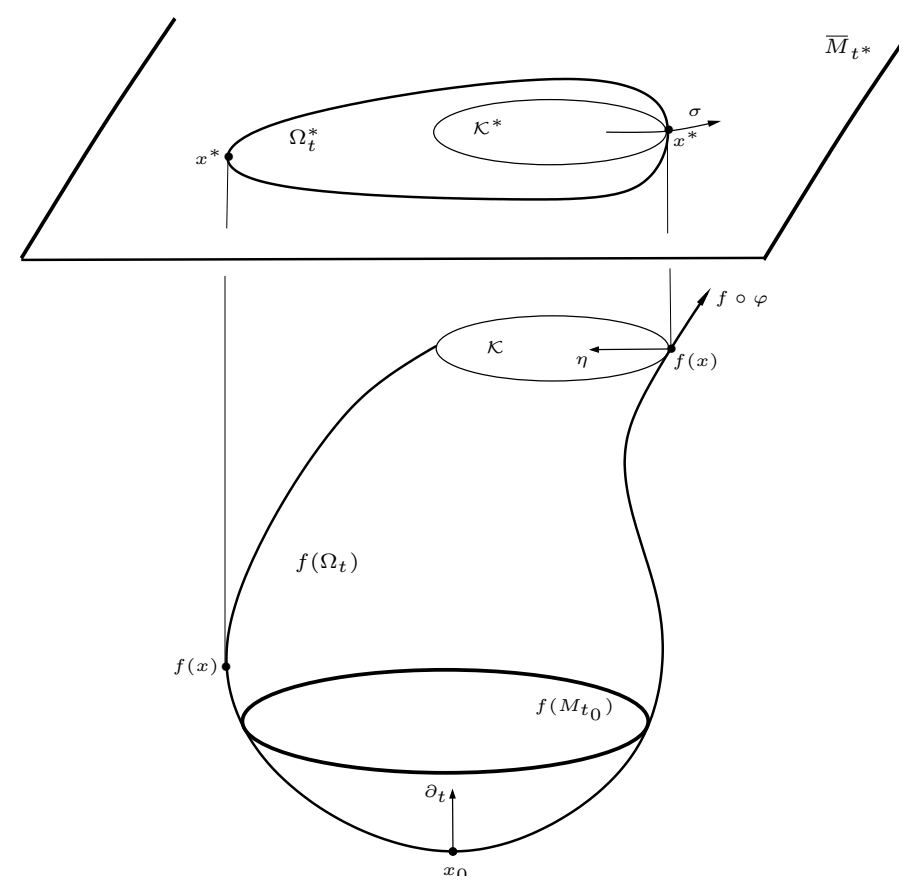

Figure 5. Projection of $f\left(\Omega_{t}\right)$ to $\bar{M}_{t^{*}}$

Let $\varphi:[0, T+\delta] \rightarrow M, \delta>0$, be an arclength parametrization of the trajectory of $\operatorname{grad} \xi$ through $x$ satisfying $\varphi(0) \in M_{t_{0}}$ and $\varphi(T)=x$. Write

$$
\varphi^{\prime}(s)=a(s) \eta(\varphi(s))+b(s) \partial_{t}, s \in[0, T+\delta],
$$


where $\eta$ is defined as in (18). We remark that $b$ is a positive function, since $\varphi^{\prime}$ is parallel to $\operatorname{grad} \xi$. Also, it is easily seen that, along $\varphi, N=b \eta-a \partial_{t}$. So,

$$
a(s)=-\Theta(\varphi(s)), s \in[0, T+\delta] .
$$

From this, equality (14), and inequality (17), we have

(19) $a^{\prime}=\left\langle A \operatorname{grad} \xi, \varphi^{\prime}\right\rangle+\phi \Theta\left\langle\operatorname{grad} \xi, \varphi^{\prime}\right\rangle=\|\operatorname{grad} \xi\|\left(\left\langle A \varphi^{\prime}, \varphi^{\prime}\right\rangle+\phi \Theta\left\langle\varphi^{\prime}, \varphi^{\prime}\right\rangle\right)>0$,

which implies that $a$ is increasing.

Let us show now that $a(T) \leq 0$. Indeed, if $x=\varphi(T) \in \Omega_{t}$, as we know, $a(T)=$ $-\Theta(x)=0$. So, we can assume $x \in \partial \Omega_{t}=M_{t}$ and $a(T) \neq 0$. In this case, denoting by $\mathcal{K}$ the convex set bounded by $f\left(M_{t}\right)$ in $\bar{M}_{t}$, and setting $\mathcal{K}^{*}:=\pi(\mathcal{K}) \subset \bar{M}_{t^{*}}$, we have that $x^{*} \in \partial \Omega_{t}^{*} \cap \partial \mathcal{K}^{*}$. Clearly, $\mathcal{K}^{*}$ is compact, and $\pi_{*} \eta(x)$ is orthogonal to $\partial \mathcal{K}^{*}$, pointing inward $\mathcal{K}^{*}$. Furthermore, $f\left(\operatorname{cl} \Omega_{t}\right)$ separates $\bar{M}^{n} \times[0, t]$, for $\left.f\right|_{\mathrm{cl} \Omega_{t}}$ is an embedding. Hence, a horizontal geodesic through any point in $\mathcal{K}^{*}$ necessarily reaches $f\left(\Omega_{t}\right)$, which yields $\mathcal{K}^{*} \subset \Omega_{t}^{*}$ (see Fig. 5).

Consider the projection $\sigma(s):=\pi(f(\varphi(s)))$ of $f \circ \varphi$ to $\bar{M}_{t^{*}}$. Since $\sigma^{\prime}(T)=$ $a(T) \pi_{*} \eta(x) \neq 0$, one has that $\sigma$ is transversal to $\partial \mathcal{K}^{*}$ (and then to $\partial \Omega_{t}^{*}$ ) at $\sigma(T)=$ $x^{*}$. Therefore, the part of $\sigma$ inside (respectively, outside) $\mathcal{K}^{*}$ is the projection of a part of $f \circ \varphi$ inside (respectively, outside) $f\left(\Omega_{t}\right)$, that is, $\sigma(s) \in \mathcal{K}^{*}$ for all small $s<T$ and $\sigma(s) \notin \mathcal{K}^{*}$ for $s>T$. This gives that the velocity vector $\sigma^{\prime}(T)$ points outward $\mathcal{K}^{*}$ at $\sigma(T)=x^{*}$, that is, $0>\left\langle\sigma^{\prime}(T), \pi_{*} \eta(x)\right\rangle=a(T)$, as claimed.

Since $a$ is increasing and both $a(0)$ and $a(T)$ are non positive, we have that $a \leq 0$ in $[0, T]$. But $a^{2}+b^{2}=1$. Hence, $a a^{\prime}+b b^{\prime}=0$, which implies that $b^{\prime} \geq 0$ in $[0, T]$, for $b>0$. Therefore, $b$ is nondecreasing. So, if we set

$$
\lambda:=\inf _{M_{t_{0}}}\left\langle\frac{\operatorname{grad} \xi}{\|\operatorname{grad} \xi\|}, \partial_{t}\right\rangle,
$$

we have that $b=\left\langle\varphi^{\prime}, \partial_{t}\right\rangle \geq \lambda>0$. Thus,

$t-t_{0}=\xi(\varphi(T))-\xi(\varphi(0))=\int_{0}^{T}(\xi \circ \varphi)^{\prime}(s) d s=\int_{0}^{T}\left\langle\operatorname{grad} \xi, \varphi^{\prime}\right\rangle d s=\int_{0}^{T} b(s) d s \geq T \lambda$, and so, the following inequalities hold:

$$
T \leq \frac{t-t_{0}}{\lambda}<\frac{t^{*}-t_{0}}{\lambda}
$$

Now, set $t(s):=\xi(\varphi(s)), s \in[0, T+\delta]$, and notice that

$$
1=\|\eta(\varphi(s))\|=\varrho(t(s))\|\eta(\varphi(s))\|_{\bar{M}} .
$$

Since $\sigma^{\prime}(s)=a(s) \pi_{*} \eta(\varphi(s))$ and $\|\eta(\varphi(s))\|_{\bar{M}}=\left\|\pi_{*} \eta(\varphi(s))\right\|_{\bar{M}}$, we have

$$
\left\|\sigma^{\prime}(s)\right\|=\varrho\left(t^{*}\right)|a(s)|\|\eta(\varphi(s))\|_{\bar{M}}=\frac{\varrho\left(t^{*}\right)}{\varrho(t(s))}|a(s)| \leq \frac{\varrho\left(t^{*}\right)}{\mu}|a(s)|,
$$

where $\mu=\left.\inf \varrho\right|_{\left[t_{0}, t^{*}\right]}$.

Therefore, denoting the length of $\sigma$ from 0 to $T$ by $\mathcal{L}(\sigma)$, considering (20), and taking into account that $a=\sqrt{1-b^{2}} \leq \sqrt{1-\lambda^{2}}$, one has

$$
\mathcal{L}(\sigma)=\int_{0}^{T}\left\|\sigma^{\prime}(s)\right\| d s \leq \frac{\varrho\left(t^{*}\right)}{\mu} \int_{0}^{T}|a(s)| d s<\frac{\varrho\left(t^{*}\right)\left(t^{*}-t_{0}\right)}{\lambda \mu} \sqrt{1-\lambda^{2}} .
$$

It follows that each point $x^{*} \in \partial \Omega_{t}^{*}$ can be joined to a point of $\pi\left(f\left(\Omega_{t_{0}}\right)\right)$ by a curve whose length is bounded by a constant independent of $t$, which clearly implies that $\Omega_{t}^{*}=\pi\left(f\left(\Omega_{t}\right)\right)$ is uniformly bounded. Consequently, $\pi(f(\Omega))$ is 
bounded in $\bar{M}_{t^{*}}$. In particular, $M_{t^{*}}$ is bounded, and so is compact, as we wished to show. This fact, as we pointed out, leads to a contradiction and then finishes the proof of the theorem.

Remark 4. Under the conditions of Theorem 4 consider the case when $\bar{M}^{n}$ is a Hadamard manifold $\mathscr{H}^{n}$, and assume further that the warp function $\varrho$ is convex, that is, $\varrho^{\prime \prime} \geq 0$. Then, in addition to the conclusions of the theorem, one has:

a) $f(M)$ bounds a convex set in $\mathbb{R} \times x_{\varrho} \mathscr{H}^{n}$.

b) When $M$ is homeomorphic to $\mathbb{R}^{n}, f(M)$ is an unbounded horizontal geodesic graph over an open set of a vertical section of $\mathbb{R} \times \varrho \mathscr{H}^{n}$.

Indeed, by [5, Theorem 7.5] (see also [5, Remark 7.7]), the convexity of $\varrho$ implies that $\mathbb{R} \times \varrho \mathscr{H}^{n}$ is a Hadamard manifold. This fact, as can be easily seen, allows us to mimic the first part of the proof of Theorem 1 and, then, get (a) and (b).

Proof of Theorem 5. As in the proof of Theorem 3, it follows from the compacity of the vertical sections of $\mathbb{R} \times{ }_{\varrho} \mathbb{S}^{n}$, the properness of $f$, and the absence of critical points of its height function that there exists a family of vertical sections,

$$
f_{t}: M_{t} \subset M \rightarrow S_{t}:=\{t\} \times \varrho \mathbb{S}^{n}, t \in \mathbb{R}
$$

such that $M=\bigcup M_{t}$, and $M_{t}$ is compact and connected for all $t \in \mathbb{R}$.

Also, by Lemma 3 and do Carmo-Warner Theorem, for all $t \in \mathbb{R}, M_{t}$ is homeomorphic to $\mathbb{S}^{n-1}, f_{t}$ is an embedding, and $f\left(M_{t}\right)$ bounds a convex set in $S_{t}$. Thus, the hypersurface $f$ itself is an embedding and $M$ is homeomorphic to $\mathbb{S}^{n-1} \times \mathbb{R}$.

Let us assume now that $M$ has nonnegative sectional curvature. Since $M$ is noncompact and not homeomorphic to $\mathbb{R}^{n}$, Perelman Soul Theorem gives that, for all $x \in M$, there exist orthonormal vectors $X, Y \in T_{x} M$ satisfying $K(X, Y)=0$. Then, considering equality 16 , we have

$$
\begin{aligned}
0 & =\left(\frac{1}{\varrho^{2}}-\phi^{2}\right)+\frac{\mathrm{L}(\varrho)-1}{\varrho^{2}}\left\|\pi_{X Y} \operatorname{grad} \xi\right\|^{2}+\operatorname{det} A_{X Y} \\
& \geq \frac{1}{\varrho^{2}}+\frac{\mathrm{L}(\varrho)-1}{\varrho^{2}}\left\|\pi_{X Y} \operatorname{grad} \xi\right\|^{2},
\end{aligned}
$$

for $\operatorname{det} A_{X Y} \geq \phi^{2}$, by the $\phi$-convexity of $f$ (see $(9)$ ).

Therefore, the inequality

$$
(1-\mathrm{L}(\varrho))\left\|\pi_{X Y} \operatorname{grad} \xi\right\|^{2} \geq 1
$$

holds and yields $\mathrm{L}(\varrho) \leq 0$, since $\left\|\pi_{X Y} \operatorname{grad} \xi\right\| \leq 1$.

From (21), we also have that $\|\operatorname{grad} \xi\|=1$ if $\mathrm{L}(\varrho)=0$ on $\mathbb{R}$. In this case, $\operatorname{grad} \xi=\partial_{t}$ on all of $M$. In particular, $\left\langle N, \partial_{t}\right\rangle=0$. Differentiating this equality and considering 13 , we easily conclude that $A \partial_{t}=0$. This, together with the $\phi$-convexity of $f$, implies that $\phi=0$ on $\mathbb{R}$ and, then, that $\varrho$ is constant.

In conclusion, we point out the following property of $\phi$-convex hypersurfaces:

Proposition 1. If $f: M^{n} \rightarrow \mathbb{R} \times{ }_{\varrho} \mathbb{Q}_{\epsilon}^{n}$ is $\phi$-convex, $M$ has sectional curvature $K \leq$ $\epsilon / \varrho^{2}$, and $\mathrm{L}(\varrho)>\epsilon$ on $M$, then each connected component of $f(M)$ is contained in a vertical section of $\mathbb{R} \times{ }_{\varrho} \mathbb{Q}_{\epsilon}^{n}$. 
Proof. From the hypothesis on $K$ and equality (16), one has

$$
\begin{aligned}
\frac{\epsilon}{\varrho^{2}} & \geq\left(\frac{\epsilon}{\varrho^{2}}-\phi^{2}\right)+\frac{\mathrm{L}(\varrho)-\epsilon}{\varrho^{2}}\left\|\pi_{X Y} \operatorname{grad} \xi\right\|^{2}+\operatorname{det} A_{X Y} \\
& \geq \frac{\epsilon}{\varrho^{2}}+\frac{\mathrm{L}(\varrho)-\epsilon}{\varrho^{2}}\left\|\pi_{X Y} \operatorname{grad} \xi\right\|^{2},
\end{aligned}
$$

which implies that $\pi_{X Y} \operatorname{grad} \xi=0 \forall X, Y \in T M$, that is, $\operatorname{grad} \xi=0$ on $M$.

It is easily seen that Proposition 1 applies to the following type of hypersurfaces:

- $f: M^{n} \rightarrow \mathbb{R} \times_{e^{-t^{2} / 2}} \mathbb{R}^{n}$ with $K \leq 0$.

- $f: M^{n} \rightarrow \mathbb{R} \times_{\cosh (t / 2)} \mathbb{H}^{n}$ with $K \leq-1$.

Acknowledgments. We are indebted to Fernando Manfio and Ruy Tojeiro for valuable suggestions which improved some results in this paper. We would also like to thank Luis Florit for helpful conversations.

\section{REFERENCES}

[1] Abresch, U., Rosenberg H.: A Hopf differential for constant mean curvature surfaces in $\mathbb{S}^{2} \times \mathbb{R}$ and $\mathbb{H}^{2} \times \mathbb{R}$. Acta Math. $193141-174$ (2004).

[2] Aledo, J., Espinar, J., Glvez, J.: Complete surfaces of constant curvature in $\mathbb{H}^{2} \times \mathbb{R}$ and $\mathbb{S}^{2} \times \mathbb{R}$. Calc. Var. 29, 347-363 (2007).

[3] Alexander, S., Locally convex hypersurfaces of negatively curved spaces. Proc. Am. Math. Soc. 64, 321-325 (1977).

[4] Bishop, R. L.: Infinitesimal convexity implies local convexity, Indiana Univ. Math. J. 24, 169-172 (1974/75).

[5] Bishop, R. L., O'Neill, B.: Manifolds of negative curvature. Trans. Amer. Math. Soc. 145, 1-49 (1969).

[6] Cheeger, J.; Gromoll, D.: On the structure of complete manifolds of nonnegative curvature. Ann. of Math. 96, No. 3, 413-443 (1972).

[7] Cheng, X., Rosenberg, H.: Embedded positive constant r-mean curvature hypersurfaces in $M^{m} \times \mathbb{R}$. Anais da academia brasileira de cincias, 77, 183-199 (2005).

[8] Currier, R. J.: On hypersurfaces of hyperbolic space infinitesimally supported by horospheres, Trans. Am. Math. Soc. 313, 419-431 (1989).

[9] Dajczer, M., Rodrguez, L.: Rigidity of real Kähler submanifolds, Duke Math. J. 53, 211-220 (1986).

[10] Daniel, B.: Isometric immersions into $\mathbb{S}^{n} \times \mathbb{R}$ and $\mathbb{H}^{n} \times \mathbb{R}$ and applications to minimal surfaces. Trans. Amer. Math. Soc. 361, Number 12, 6255-6282 (2009).

[11] de Lima, R. F., de Andrade, R. L.: Convexity, rigidity, and reduction of codimension of isometric immersions into space forms. Bull. Braz. Math. Soc. 50, 119-136 (2019).

[12] do Carmo, M., Lima, E.: Immersions of manifolds with non-negative sectional curvatures, Bol. Soc. Brasil. Mat. 2, 9-22 (1971).

[13] do Carmo, M., Warner, F.: Rigidity and convexity of hypersurfaces in spheres, J. Diff. Geom. 4, 133-144 (1970).

[14] Espinar, J., Glvez, A.: Rosenberg, H.: Complete surfaces with positive extrinsic curvature in product spaces, Comment. Math. Helv. 84, 351-386 (2009).

[15] Espinar, J., de Oliveira, I.: Locally convex surfaces immersed in a Killing submersion, Bull Braz Math Soc, New Series, 44 (1), 155-171 (2013).

[16] Espinar, J., Rosenberg, H.: When strictly locally convex hypersurfaces are embedded, Math. Z., 271, 1075-1090 (2012) 
[17] Hadamard, J.: Sur certaines proprietés des trajectoires en dynamique, J. Math. Pures Appl. 3, 331-387 (1897).

[18] Hadamard, J.: Les surfaces à courbure opposées et leurs lignes géodesique, J. Math. Pures Appl. 4, 27-73 (1898).

[19] Heijenoort, J.: On locally convex manifolds, Comm. on pure and appl. math. 5, 223-242 (1952).

[20] Hsiang, W-T., Hsiang, W-y.: On the uniqueness of isoperimetric solutions and imbedded soap bubbles in non-compact symmetric spaces, I, Invent. math. 98, 39-58 (1989).

[21] Lawn, M-A., Ortega, M.: A fundamental theorem for hypersurfaces in semi-Riemannian warped products. J. Geom. Phys. 90, 55-70 (2015).

[22] Leandro, B., Pina, R., Santos, J. P.: Einstein hypersurfaces of $\mathbb{S}^{n} \times \mathbb{R}$ and $\mathbb{H}^{n} \times \mathbb{R}$. To appear in Bull. Braz. Math. Soc. (avaiable at https://arxiv.org/abs/1910.06930).

[23] Manfio, F., Tojeiro, R.: Hypersurfaces with constant sectional curvature of $\mathbb{S}^{n} \times \mathbb{R}$ and $\mathbb{H}^{n} \times \mathbb{R}$. Illinois J. Math. 55, 397-415 (2011).

[24] Milnor, J.: Morse Theory. Annals of Mathematics Studies (1973).

[25] Oliveira, I., Schweitzer, S.: Locally convex hypersurfaces immersed in $H^{n} \times \mathbb{R}$. Geom Dedicata 188, 17-32 (2017).

[26] O'Neill, B.: Semi-Riemannian geometry. Academic Press (1983).

[27] Pedrosa, R.: The isoperimetric problem in spherical cylinders, Ann. Glob. Anal. and Geom. 26 333-354 (2004).

[28] Perelman, T.: Proof of the soul conjecture of Cheeger and Gromoll, J. Differential Geom. 40, 209-212 (1994).

[29] Rosenberg, H., Tribuzy, R.: Rigidity of convex surfaces in the homogeneous spaces, Bull. Sci. math. 136, 892-898 (2012).

[30] Sacksteder, R.: On hypersurfaces with no negative sectional curvatures, Amer. J. Math. 82, 609-630 (1960).

[31] Sacksteder, R.: The rigidity of hypersurfaces, J. Math. Mech. 11, 929-940 (1962).

[32] Spivak, M.: A compreensive introduction to differential geometry - Vol IV, Publish or Perish (1979).

[33] Stoker, J.: Über die Gestalt der positiv gekrümmten offenen Flächen im dreidimensionalen Raume, Compositio Math. 3, 55-88 (1936).

[34] R. Tojeiro, On a class of hypersurfaces in $\mathbb{S}^{n} \times \mathbb{R}$ and $\mathbb{H}^{n} \times \mathbb{R}$, Bull. Braz. Math. Soc. 41 (2010), 199-209. 